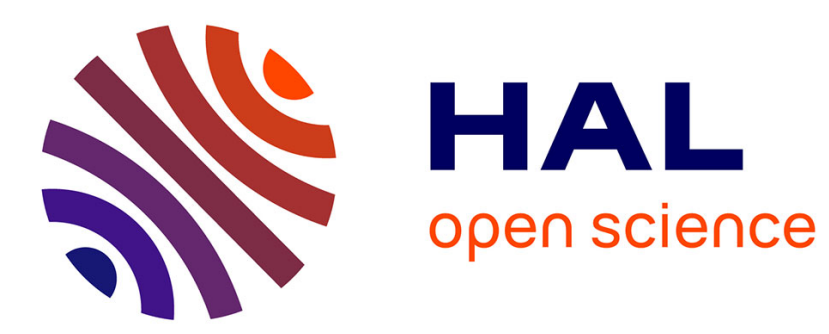

\title{
A low-diffusion self-adaptive flux-vector splitting approach for compressible flows
}

\author{
David Iampietro, Frédéric Daude, Pascal Galon
}

\section{To cite this version:}

David Iampietro, Frédéric Daude, Pascal Galon. vector splitting approach for compressible flows. 10.1016/j.compfluid.2020.104586 . hal-01945480v2

A low-diffusion self-adaptive fluxComputers and Fluids, 2020, 206(C),

HAL Id: hal-01945480

https://hal.science/hal-01945480v2

Submitted on 18 Dec 2018

HAL is a multi-disciplinary open access archive for the deposit and dissemination of scientific research documents, whether they are published or not. The documents may come from teaching and research institutions in France or abroad, or from public or private research centers.
L'archive ouverte pluridisciplinaire HAL, est destinée au dépôt et à la diffusion de documents scientifiques de niveau recherche, publiés ou non, émanant des établissements d'enseignement et de recherche français ou étrangers, des laboratoires publics ou privés. 


\title{
A low-diffusion self-adaptive flux-vector splitting approach for compressible flows
}

\author{
D. Iampietro ${ }^{\mathrm{a}, \mathrm{b}, 1, *}$, F. Daude $\mathrm{D}^{\mathrm{a}, \mathrm{b}}$, P. Galon ${ }^{\mathrm{b}, \mathrm{c}}$ \\ ${ }^{a}$ EDF RED, Département ERMES, 7 Boulevard Gaspard Monge, 91120 Palaiseau, France \\ ${ }^{b}$ IMSIA, UMR EDF/CNRS/CEA/ENSTA 9219, Université Paris-Saclay, 828 Boulevard des Maréchaux, 91762 Palaiseau Cedex, France \\ ${ }^{c} C E A, D E N, D A N S, D M 2 S$, SEMT, DYN, F-91191 Gif-sur-Yvette, France \\ ${ }^{d}$ I2M, UMR CNRS 7373, Technopôle Château-Gombert, 39 rue F. Joliot Curie, F-13453 Marseille, France
}

\begin{abstract}
A low-diffusion self-adaptive flux-vector splitting method is presented for the Euler equations. The flux-vector is here split into convective and acoustic parts following the formulation recently proposed by the authors. This procedure is based on the Zha-Bilgen (or previously Baraille $e t$ al. for the Euler barotropic system) approach enriched by a dynamic flow-dependent splitting parameter based on the local Mach number. As a consequence, in the present self-adaptive splitting, the convective and acoustic parts decouple in the low-Mach number regime whereas the complete Euler equations are considered for the sonic and highly subsonic regimes. The low diffusive property of the present scheme is obtained by adding anti-diffusion terms to the momentum and the energy components of the pressure flux in the acoustic part of the present splitting. This treatment results from a formal invariance principle preserving the discrete incompressible phase space through the pressure operator. Numerical results for several carefully chosen one- and two-dimensional test problems are finally investigated to demonstrate the accuracy and robustness of the proposed scheme for a wide variety of configurations from subsonic to highly subsonic flows.
\end{abstract}

Keywords: Euler equations, Flux-Vector Splitting, Low-Mach number flows, Stationary incompressible flows, Operator kernel

\section{Introduction}

The objective of the present work is to provide a time-explicit numerical scheme able to capture fast acoustic waves related to the compressible Euler system while remaining accurate, when the flow Mach number tends towards zero, with stationary solutions of the incompressible Euler system. Although the development of accurate methods for the Euler and Navier-Stokes equations is the focus of numerous research studies over the last few decades, fulfilling both criteria is still challenging. Indeed, upwind schemes are known to be well suited for the solution of hyperbolic conservation laws, and are thus extensively used to solve the compressible Euler equations $[1,2,3]$. These approaches can broadly be divided into two main categories: Godunov-type methods and Flux-Vector Splitting (FVS) methods.

Godunov-type methods are based on the approximate solution of the local Riemann problem between two adjacent states for the calculation of the numerical flux through the interface between these two states. Complete Riemann solvers as the Roe's approximate Riemann solver [4] or the HLLC (Harten-Lax-van Leer + Contact) scheme of Toro $[5,3]$, respect the full wave structure of the compressible Euler equations in the construction of the numerical flux. As a consequence, these solvers are known for their accuracy and for their ability to capture both linearly degenerate and genuinely non-linear waves. Generally, this family of methods is usually complex and computationally expensive.

In Flux-Vector Splitting methods, the flux is usually split into positive and negative components as in the pioneer work of Steger and Warming [6]. These techniques are usually less expensive than Godunov-type methods and provide an efficient upwind discretisation. However their inability to resolve intermediate characteristic fields badly affects

${ }^{*}$ Corresponding author: david60.iampietro@gmail.com 
the correct resolution of contact waves, material interfaces, shear waves and vortices for example. To circumvent this problem, Liou and Steffen [7] have proposed a flux-vector splitting named AUSM (Advection Upstream Splitting Method) able to recognize contact waves. The basic idea of AUSM-type solvers is to split the flux-vector into its convective and acoustic parts at the continuous level. Subsequently, both parts are treated separately based on their respective characteristic fields. Other strategies have been proposed to separate the convective and acoustic parts of the flux as the one from Zha and Bilgen [8] or more recently from Toro and Vazquez [9] and from Mandal and Panwar [10]. Contact preservation is achieved by modifying the dissipation vector of the pressure flux using isentropic relations [10].

Though upwind methods can handle the waves related to the compressible Euler system, it is known that they fail to be accurate with respect to solutions of the asymptotically incompressible Euler system when the Mach number tends to zero. Such a drawback has been extensively studied in the literature. The pioneering work of Guillard and Viozat [11], based on a formal asymptotic decomposition of the discrete solution obtained using the Roe's scheme, has formally revealed that, a non-dimensional pressure term scaling as $O(M)$ is inherently present in the discrete solution. In [12], Guillard and Murrone inject a Mach power expansion inside the exact solution of a Riemann problem related to the isentropic Euler system. They show that the spurious pressure term of order $O(M)$ corresponds to an artificial viscosity stemming from the normal momentum equation of the acoustic part of the system. Subsequently, the presence of such a numerical diffusion has been discovered in [13] for a large number of approximate Riemann solvers. Enhancing the understanding of the low Mach number issue, the work of Dellacherie [14] and Dellacherie et al. [15] establishes a link between the above artificial acoustic viscosity and the disturbance of the discrete incompressible phase space throughout time. Indeed, by studying the modified equation obtained from an exact Godunov scheme related to the linear wave system, the authors prove that the presence of the acoustic viscosity in the momentum equation "shrinks" the kernel associated with the acoustic operator. As a consequence, the latter kernel is strictly included in the incompressible phase space. This means that after one time-step, initial conditions belonging to the discrete incompressible space might be disturbed by the discrete acoustic operator. As highlighted in [16], this issue disappears in 1-D or in multi-D if a triangular (tetrahedral) mesh is used. In order to partly solve this difficulty, many works $[17,18,15,19,20]$ propose to weaken the order of the artificial viscosity in the normal momentum equation by multiplying it with a term proportional to the local Mach number. The same procedure is applied to the AUSM scheme leading to the $\mathrm{AUSM}^{+}$-up method [21, 22]. In terms of stability, the consequences of the above artificial viscosity reduction, depend on the numerical method at stake. Indeed, in [15] a linear $L^{2}$-stability study shows that Godunov-like solvers remain a priori stable under a classical CFL condition based on the biggest Euler eigenvalues. On the contrary, the FVS-type AUSM+r-up is binded by an extremely severe explicit stability constraint.

In the current contribution, the proposed numerical scheme combines the simplicity associated with the FVS methods while keeping a good control on the acoustic operator discretization. Its formulation relies on a convective/acoustic decomposition originally proposed by Zha, Bilgen [8] and Baraille [23] for the complete Euler system and by Baraille et al. [24] for the Euler barotropic system. The convective part is evaluated via a simple upwinding. The acoustic part is discretized using a HLLC-like Riemann solver. By doing so, one can easily identify, in the normal contribution of the momentum flux, the artificial viscosity term responsible for the low Mach number accuracy issue. Besides, a formal analysis, based on the sufficient criterion related to the discrete incompressible space invariance through the application of the discrete acoustic operator is presented here. It suggests that additional low-Mach corrections should be also included inside the energy flux of the acoustic part.

In order to fully distinguish the numerical treatment of the present FVS scheme in sonic flow regimes from the subsonic ones, a dynamic flow-dependent splitting parameter is introduced. As described recently in [25], such a splitting parameter acts as a weighting factor between the convective and the acoustic part of the flux. Its definition is based on the local Mach number such as for low-Mach configurations the convective and acoustic parts of the flux completely decouple whereas for sonic and highly subsonic configurations, the complete Euler equations are retrieved in the convective part of the considered splitting. This makes the present numerical scheme self-adaptive as the low-Mach regions can be detected in both space and time.

The paper is organized as follows. Section 2 is devoted to the governing equations associated to the Euler system. The numerical approaches considered in the present work are then presented in Section 3, in particular the HLLC scheme and the present self-adaptive splitting. Afterwards, focus is given on the construction of low Mach corrections in Section 4. The classical reduction of the numerical diffusion in the momentum equation [14] is recalled and a special formal analysis is carried out on the pressure flux in the acoustic part issued from the present splitting. Three 
numerical methods, HLLC, HLLC with a standard low-Mach correction and the present FVS approach, are then assessed on several problems in Section 5. The numerical tests involve highly subsonic configurations, stationary incompressible test cases as well as simulations where fast acoustic waves and low-Mach number solutions interact.

\section{Governing equations}

The set of compressible Euler equations is here considered for inviscid compressible flows:

$$
\partial_{t} \boldsymbol{U}+\nabla \cdot \boldsymbol{F}(\boldsymbol{U})=\mathbf{0} \quad \text { with } \quad \boldsymbol{U}=\left(\begin{array}{c}
\rho \\
\rho \boldsymbol{u} \\
\rho e
\end{array}\right) \quad \text { and } \quad \boldsymbol{F}(\boldsymbol{U})=\left(\begin{array}{c}
\rho \boldsymbol{u} \\
\rho \boldsymbol{u} \otimes \boldsymbol{u}+p \boldsymbol{I}_{d} \\
(\rho e+p) \boldsymbol{u}
\end{array}\right)
$$

where $\rho, \boldsymbol{u}, p$ and $e$ are respectively the density, the velocity vector, the pressure, the specific total energy and $\boldsymbol{I}_{d}$ the identity tensor. The specific internal energy $\varepsilon$ is given by: $\varepsilon=e-\boldsymbol{u}^{2} / 2$. The set of Eq. (1) is completed by an equation of state (EOS): $\varepsilon=\varepsilon(\rho, p)$. The speed of sound denoted by $c$ is obtained via the EOS: $c^{2} \equiv\left(p / \rho-\partial_{\rho} \varepsilon\right)\left(\partial_{p} \varepsilon\right)^{-1}$. Let us mention that the formulation of the numerical scheme proposed below is independent of the EOS. However, the applications presented here only involve ideal gas EOS for which: $\rho \varepsilon=p /(\gamma-1)$ and $c=\sqrt{\gamma p / \rho}$; $\gamma$ being the heat capacity ratio.

The model in Eq. (1) is known to be hyperbolic and admits three eigenvalues in 1-D: $\lambda_{1}=u-c, \lambda_{2}=u$ and $\lambda_{3}=u+c$. The field associated with eigenvalue $\lambda_{2}$ is linearly degenerate (LD), other fields are genuinely non-linear (GNL).

In the sequel, the framework related to unstructured multi-D Finite-Volume schemes is introduced. Different numerical fluxes are also described.

\section{Numerical approach}

The present scheme relies on the integral form of Eq. (1):

$$
\frac{\mathrm{d}}{\mathrm{d} t} \int_{C} \boldsymbol{U} \mathrm{d} \boldsymbol{V}+\int_{\partial C} \boldsymbol{F}(\boldsymbol{U}) \boldsymbol{n} \mathrm{d} \boldsymbol{S}=\mathbf{0},
$$

where $C$ is the control volume, $\partial C$ its boundary whose unit outward normal vector is denoted by $\boldsymbol{n}$; the cell-centered Finite-Volume discretisation can be written as:

$$
\boldsymbol{U}_{i}^{n+1}=\boldsymbol{U}_{i}^{n}-\frac{\Delta t^{n}}{\left|C_{i}\right|} \sum_{j \in V(i)} \boldsymbol{\Phi}_{i, j}\left|S_{i, j}\right|
$$

with $\boldsymbol{U}_{i}^{n}$ the cell average of the conservative variables vector $\boldsymbol{U}$ on the cell $C_{i}$ at time $t^{n}$ and $\boldsymbol{\Phi}_{i, j}$ the time-explicit numerical flux-vector at the cell interface $S_{i, j}$ between the cell $C_{i}$ and the cell $C_{j} . V(i)$ refers to the neighboring cells of $C_{i}$. Note that, in Eq. (3), $\left|C_{i}\right|$ stands for the volume of the cell $C_{i}$ whereas $\left|S_{i, j}\right|$ is the surface of the interface between $C_{i}$ and $C_{j}$. The time step $\Delta t^{n}$ used in Eq. (3) is given by the Courant number $C$ defined as:

$$
C=\Delta t^{n} \max _{i}\left(\max _{j \in V(i)}\left(\frac{\left|\boldsymbol{u}_{i}^{n} \cdot \boldsymbol{n}_{i, j}\right|+c_{i}^{n}}{h_{i}}\right)\right),
$$

with $h_{i}$ the characteristic length of the cell $C_{i}$ and $\boldsymbol{n}_{i, j}$ the outward (from $C_{i}$ to $C_{j}$ ) unit normal vector of a given interface $S_{i, j}$. Several numerical flux-vectors are considered in the following. Without loss of generality the numerical fluxes are written using a 2-D framework. 


\subsection{HLLC scheme}

Consider the one-dimensional Riemann problem related to system (1) projected on the direction pointed by the normal $\boldsymbol{n}_{i, j}$. Besides, define $\boldsymbol{t}_{i, j}$, the tangential unit vector related to $\boldsymbol{n}_{i, j}$. Along the normal direction, let us introduce the left and right states of the interface denoted by $\boldsymbol{U}_{L}$ and $\boldsymbol{U}_{R}$ respectively.

The HLLC (Harten, Lax, van-Leer + Contact) scheme [5, 3] is an approximate Riemann solver considering all the waves of the Euler equations. Its numerical flux writes as:

$$
\boldsymbol{\Phi}_{i, j}^{\mathrm{HLLC}}= \begin{cases}\boldsymbol{\Phi}_{L} & \text { if } 0<S_{L}, \\ \boldsymbol{\Phi}_{L}^{*} & \text { if } S_{L} \leq 0<S_{M}, \\ \boldsymbol{\Phi}_{R}^{*} & \text { if } S_{M} \leq 0<S_{R}, \\ \boldsymbol{\Phi}_{R} & \text { if } S_{R} \leq 0,\end{cases}
$$

where $\boldsymbol{\Phi}_{K}=\boldsymbol{F}\left(\boldsymbol{U}_{K}\right) \boldsymbol{n}_{i, j}$ with the flux-vector

$$
\boldsymbol{F}(\boldsymbol{U}) \boldsymbol{n}=\left(\begin{array}{c}
\rho \boldsymbol{u} \cdot \boldsymbol{n} \\
\rho \boldsymbol{u} \cdot \boldsymbol{n}+p \boldsymbol{n} \\
(\rho e+p) \boldsymbol{u} \cdot \boldsymbol{n}
\end{array}\right)
$$

and

$$
\boldsymbol{\Phi}_{K}^{*}=\boldsymbol{\Phi}_{K}+S_{K}\left(\boldsymbol{U}_{K}^{*}-\boldsymbol{U}_{K}\right)
$$

with,

$$
\boldsymbol{U}_{K}^{*}=\rho_{K} \frac{\left(S_{K}-\boldsymbol{u}_{K} \cdot \boldsymbol{n}_{i, j}\right)}{\left(S_{K}-S_{M}\right)}\left(\begin{array}{c}
1 \\
S_{M} \\
\boldsymbol{u}_{K} \cdot \boldsymbol{t}_{i, j} \\
\rho_{K} e_{K}+\left(S_{M}-\boldsymbol{u}_{K} \cdot \boldsymbol{n}_{i, j}\right)\left(S_{M}+\frac{p_{K}}{\rho_{K}\left(S_{K}-\boldsymbol{u}_{K} \cdot \boldsymbol{n}_{i, j}\right)}\right)
\end{array}\right) \text { where } \quad K=L, R .
$$

The pressure $p_{M}$ is given by:

$$
p_{M}=\rho_{L}\left(S_{L}-\boldsymbol{u}_{L} \cdot \boldsymbol{n}_{i, j}\right)\left(S_{M}-\boldsymbol{u}_{L} \cdot \boldsymbol{n}_{i, j}\right)+p_{L}=\rho_{R}\left(S_{R}-\boldsymbol{u}_{R} \cdot \boldsymbol{n}_{i, j}\right)\left(S_{M}-\boldsymbol{u}_{R} \cdot \boldsymbol{n}_{i, j}\right)+p_{R},
$$

and the speed $S_{M}$ is defined as:

$$
S_{M}=\frac{\rho_{R} \boldsymbol{u}_{R} \cdot \boldsymbol{n}_{i, j}\left(S_{R}-\boldsymbol{u}_{R} \cdot \boldsymbol{n}_{i, j}\right)-\rho_{L} \boldsymbol{u}_{L} \cdot \boldsymbol{n}_{i, j}\left(S_{L}-\boldsymbol{u}_{L} \cdot \boldsymbol{n}_{i, j}\right)+p_{L}-p_{R}}{\rho_{R}\left(S_{R}-\boldsymbol{u}_{R} \cdot \boldsymbol{n}_{i, j}\right)-\rho_{L}\left(S_{L}-\boldsymbol{u}_{L} \cdot \boldsymbol{n}_{i, j}\right)} .
$$

The speed $S_{L}$ and $S_{R}$ corresponding to the fastest waves at each side of the interface are computed as proposed in Batten et al. [26]:

$$
S_{L}=\min \left(\boldsymbol{u}_{L} \cdot \boldsymbol{n}_{i, j}-c_{L}, \hat{\boldsymbol{u}} \cdot \boldsymbol{n}_{i, j}-\hat{c}\right) \quad \text { and } \quad S_{R}=\max \left(\boldsymbol{u}_{R} \cdot \boldsymbol{n}_{i, j}+c_{R}, \hat{\boldsymbol{u}} \cdot \boldsymbol{n}_{i, j}+\hat{c}\right),
$$

with $\hat{\boldsymbol{u}}$ and $\hat{c}$ the Roe average [4] of the velocity $\boldsymbol{u}$ and speed of sound $c$ variables defined as:

$$
\hat{f}=\frac{\sqrt{\rho_{L}} f_{L}+\sqrt{\rho_{R}} f_{R}}{\sqrt{\rho_{L}}+\sqrt{\rho_{R}}}
$$

This numerical scheme was previously used in two-dimensional Euler equations [5], as well as in steady supersonic 


\subsection{Flux-vector splitting approach}

Based on the work of Iampietro et al. [25, 30], the flux-vector is split into a convective part and a pressure part as:

$$
\boldsymbol{F}(\boldsymbol{U}) \boldsymbol{n}=\boldsymbol{C}(\boldsymbol{U}) \boldsymbol{n}+\left(1-\mathscr{E}_{0}^{2}\right) \boldsymbol{P}(\boldsymbol{U}) \boldsymbol{n} \quad \text { with } \quad \boldsymbol{C}(\boldsymbol{U}) \boldsymbol{n}=\boldsymbol{U} \boldsymbol{u} \cdot \boldsymbol{n}+\mathscr{E}_{0}^{2} \boldsymbol{P}(\boldsymbol{U}) \boldsymbol{n} \quad \text { and } \quad \boldsymbol{P}(\boldsymbol{U}) \boldsymbol{n}=\left(\begin{array}{c}
0 \\
p \boldsymbol{n} \\
p \boldsymbol{u} \cdot \boldsymbol{n}
\end{array}\right) \text {, }
$$

where $\mathscr{E}_{0}$ is a dynamic flow-dependent splitting parameter which is detailed in the following. Note that the specific choice $\mathscr{E}_{0}=0$ corresponds to the splitting proposed by Baraille et al. in [24] for the Euler barotropic system and by Zha and Bilgen in [8] for the whole Euler system. Both convective and acoustic subsystems issued from the present splitting have been theoretically studied in [25]. The pressure part of the flux-vector is evaluated based on the acoustic solver [3]. Its expression is similar to the one of a HLLC-type solver related to the system:

$$
\partial_{t} \boldsymbol{U}+\nabla \cdot \boldsymbol{P}(\boldsymbol{U})=\mathbf{0} .
$$

It simply writes:

$$
\boldsymbol{\Pi}_{i, j}=\left(\begin{array}{c}
0 \\
p^{*} \boldsymbol{n}_{i, j} \\
p^{*} u^{*}
\end{array}\right) \text { with }\left\{\begin{aligned}
p^{*} & =\frac{\mathcal{S}_{L} p_{R}+\mathcal{S}_{R} p_{L}-\mathcal{S}_{R} \mathcal{S}_{L}\left(\boldsymbol{u}_{R} \cdot \boldsymbol{n}_{i, j}-\boldsymbol{u}_{L} \cdot \boldsymbol{n}_{i, j}\right)}{\mathcal{S}_{R}+\mathcal{S}_{L}}, \\
u^{*} & =\frac{\mathcal{S}_{R} \boldsymbol{u}_{R} \cdot \boldsymbol{n}_{i, j}+\mathcal{S}_{L} \boldsymbol{u}_{L} \cdot \boldsymbol{n}_{i, j}-\left(p_{R}-p_{L}\right)}{\mathcal{S}_{R}+\mathcal{S}_{L}},
\end{aligned}\right.
$$

with for $K=L, R$, the acoustic impedance $\mathcal{S}_{K}$ being defined as:

$$
\mathcal{S}_{K}=\rho_{K} c_{K} .
$$

In Figure 1, the wave structure as well as the intermediate states associated with the HLLC approximate solver of

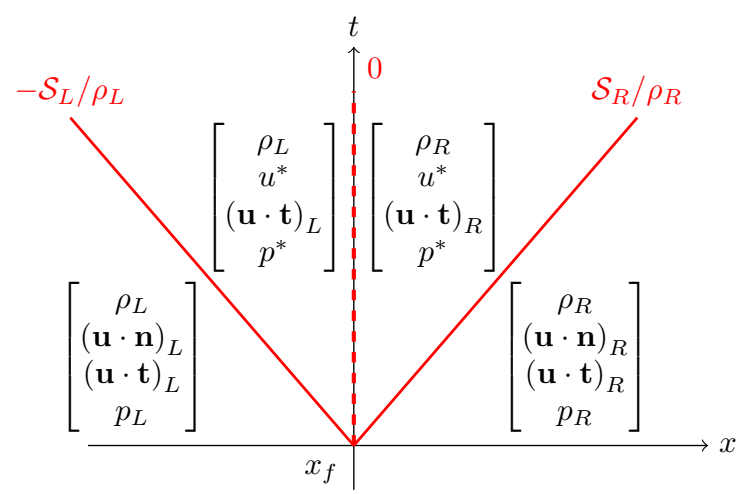

Figure 1: HLLC approximate Riemann solver for the pressure system: waves and states.

system (14) are displayed. Let us mention that, once again, $\mathbf{t}$ stands for the tangential unit vector orthogonal to $\mathbf{n}$ in a 2-D unstructured mesh.

The convective part of the flux-vector is then evaluated using the following upwinding:

$$
\boldsymbol{\Gamma}_{i, j}=\frac{1}{2}\left[u^{*} \boldsymbol{W}_{R}+u^{*} \boldsymbol{W}_{L}-\left|u^{*}\right|\left(\boldsymbol{W}_{R}-\boldsymbol{W}_{L}\right)\right]+\mathscr{E}_{0}^{2}\left(\begin{array}{c}
0 \\
p^{*} \boldsymbol{n}_{i, j} \\
0
\end{array}\right),
$$

with for $K=L, R$,

$$
\boldsymbol{W}_{K}=\left(\begin{array}{c}
\rho_{K} \\
(\rho \boldsymbol{u})_{K} \\
(\rho e)_{K}+\mathscr{E}_{0}^{2} p_{K}
\end{array}\right)
$$


As it can be noticed, the upwinding leans on the intermediate velocity $u^{*}$ in the acoustic subsystem and is applied to each flux term driven by $\boldsymbol{u} \cdot \boldsymbol{n}$. As for the residual $\mathscr{E}_{0}^{2} \nabla p$ of the pressure gradient in the momentum equation, it is discretized using the intermediate acoustic pressure $p^{*}$. Finally, the numerical flux-vector is given by:

$$
\boldsymbol{\Phi}_{i, j}^{\mathrm{FVS}}=\boldsymbol{\Gamma}_{i, j}+\left(1-\mathscr{E}_{0}^{2}\right) \boldsymbol{\Pi}_{i, j}
$$

where

$$
\mathscr{E}_{0}=\max \left(\mathscr{E}_{\mathrm{inf}}, \min (1, \tilde{M})\right), \quad 0<\mathscr{E}_{\mathrm{inf}} \ll 1 .
$$

Here, $\tilde{M}$ stands for the local Mach number built using the HLLC material velocity, $S_{M}$, at cell interface $S_{i, j}$ given in Eq. (10). For example, in 2-D, one can consider $v^{*}$ such that:

$$
v^{*}= \begin{cases}\boldsymbol{u}_{L} \cdot \boldsymbol{t}_{i, j} & \text { if } 0<S_{M}, \\ \boldsymbol{u}_{R} \cdot \boldsymbol{t}_{i, j} & \text { if } 0 \geq S_{M} .\end{cases}
$$

Introducing the local speed of sound at the interface:

$$
\tilde{c}=\max \left(c_{L}, c_{R}\right)
$$

the local Mach number is then defined as:

$$
\tilde{M}=\sqrt{\left(S_{M}\right)^{2}+\left(v^{*}\right)^{2}} / \tilde{c}
$$

If one considers the underlying convective and pressure sub-systems associated with the flux decomposition (13), it is known [24] that when $\mathscr{E}_{0}=0$, the convective sub-system

$$
\partial_{t} \boldsymbol{U}+\nabla \cdot \boldsymbol{C}(\boldsymbol{U})=\mathbf{0}
$$

is no longer hyperbolic. Hence, in order to prevent issues in zero material-velocity regions, the strictly positive lower bound $\mathscr{E}_{\text {inf }}$ has been introduced in the definition of the parameter $\mathscr{E}_{0}$. In the following, $\mathscr{E}_{\text {inf }}$ is taken equal to $10^{-8}$.

After having completely defined the present numerical scheme, let us stress that the choice of $p^{*}$ to discretize the pressure gradient residual contribution in the momentum flux of $\boldsymbol{C}(\boldsymbol{U}) \boldsymbol{n}$ is crucial. Indeed, when the local Mach number of the flow goes over one, the parameter $\mathscr{E}_{0}$ tends towards one and the pressure part $\left(1-\mathscr{E}_{0}^{2}\right) \boldsymbol{P}(\boldsymbol{U}) \boldsymbol{n}$ of the splitting cancels out. Hence, in the momentum equation, the contribution related to the pressure gradient exclusively stems from the convective part of the flux. The non-centered part of $p^{*}$ then improves the stability of the present approach when highly compressible shock waves propagate through the flow.

Besides when the local Mach number tends towards zero, $\mathscr{E}_{0}$ also tends towards zero and the splitting completely decouples convection from acoustic wave production. In such a low Mach number regime, the ability of approximate Riemann solvers to be accurate with respect to the solutions of the incompressible Euler system requires to apply low-Mach corrections. In the following, this issue is briefly presented and low Mach corrections met in many works of the literature are described. Then, the low Mach correction strategy of the present approach is detailed.

\section{Low-Mach correction}

\subsection{Low-Mach correction based on the work of Dellacherie et al.}

In the very complete work of Dellacherie et al. [15], the authors study the behavior of Godunov-like schemes applied to a linear wave equation obtained from the Euler barotropic system. They prove that, for 2-D cartesian meshes, the kernel of the acoustic operator associated with the linear wave equation is strictly smaller than the discrete space related to incompressible solutions. As a consequence, after one time-step, initial solutions that belong to this discrete incompressible space might be distorted by the acoustic operator and acoustic modes might grow.

The authors then show that such a lack of accuracy is caused by the numerical diffusion of the non-centered part of the momentum flux scaling as $O(\Delta x / M)$. In addition, a simple All-Mach number cure is proposed. In the subsonic 
case, it simply consists in reducing the prohibitive momentum numerical diffusion by multiplying the non-centered part of the momentum flux by a term denoted by $\theta$ proportional to the local flow Mach number. Subsequently we will refer to this term as "low-Mach correction".

Following the work of Dellacherie et al. [15], the HLLC scheme described previously is modified for low-Mach number considerations as:

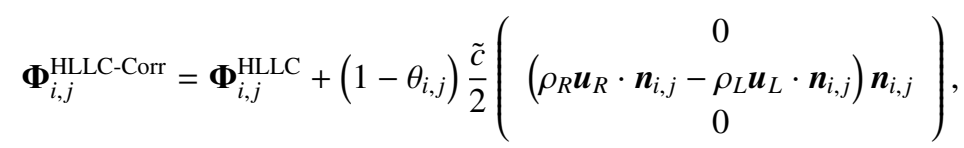

with,

$$
\theta_{i, j}=\min (1, \tilde{M})
$$

Let us mention that similar low-Mach corrections holding on the normal component related to the interface $S_{i, j}$ of the momentum equation have been extensively used in many works of the literature $[17,18,31]$.

In the next subsection, a formal analysis inspired from discrete space invariance arguments already presented in $[16,15]$ for the linear wave equation is performed. Such an analysis provides elements of justification for the different low-Mach corrections applied to the non-linear acoustic operator of the present approach. In the framework of low Mach number stationary flows, the need for an additional low-Mach correction in the energy flux is notably studied.

\subsection{Low-Mach correction of the present approach}

Consider a two-dimensional regular cartesian mesh made of $\mathrm{N}_{x} \times \mathrm{N}_{y}=N$ cells of length $\Delta x$. A part of such a mesh is displayed in Figure 2. Let us also introduce $\mathcal{I}_{N}$, the discrete space of non-dimensional solutions such that:

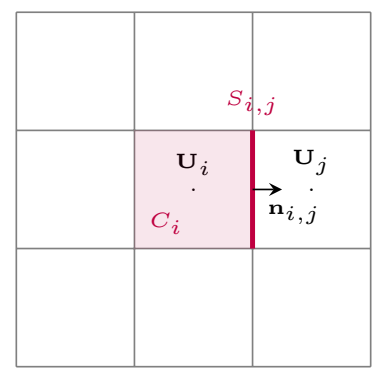

Figure 2: Two-dimensional regular cartesian mesh

$$
I_{N}=\left\{\begin{aligned}
\boldsymbol{V}_{i}=\left[\begin{array}{l}
\rho \\
\boldsymbol{u} \\
p
\end{array}\right]_{i}, i \in[1, N], \text { such that }: & \sum_{j \in V(i)} \frac{\boldsymbol{u}_{i}+\boldsymbol{u}_{j}}{2} \cdot \boldsymbol{S}_{i, j}=0, \\
& p_{i}=p_{i}^{(0),(1)}+O\left(M^{2}\right), \sum_{j \in V(i)} \frac{p_{i}^{(0),(1)}+p_{j}^{(0),(1)}}{2} \boldsymbol{S}_{i, j}=\mathbf{0} .
\end{aligned}\right\},
$$

with $\boldsymbol{S}_{i, j}=\left|S_{i, j}\right| \boldsymbol{n}_{i, j}$. Considering the above cartesian mesh, space $\mathcal{I}_{N}$ is a first-order discrete approximation of the incompressible space characterized by a divergence-free velocity field and a zero pressure gradient at zeroth and first order with respect to the Mach number. One can underline that, in [16], a more specific definition based on the Hodge decomposition of the above discrete incompressible space is given. Let us now introduce the kernel of the dimensional discrete acoustic operator $\left(1-\mathscr{E}_{0}^{2}\right) \boldsymbol{P}(\boldsymbol{U}) \boldsymbol{n}$ detailed in equations (13) and (15):

$$
\{\operatorname{Ker} \boldsymbol{P}\}_{N}=\left\{\begin{aligned}
\boldsymbol{V}_{i}=\left[\begin{array}{l}
\rho \\
\boldsymbol{u} \\
p
\end{array}\right]_{i}, i \in[1, N], \text { such that } \quad: & \sum_{j \in V(i)}\left(1-\left(\mathscr{E}_{0}\right)_{i, j}^{2}\right) p_{i, j}^{*, \theta} \boldsymbol{S}_{i, j}=\mathbf{0}, \\
& \sum_{j \in V(i)}\left(1-\left(\mathscr{E}_{0}\right)_{i, j}^{2}\right) p_{i, j}^{*, \theta} u_{i, j}^{*, \beta}\left|S_{i, j}\right|=0
\end{aligned}\right\} .
$$


with,

$$
\left\{\begin{array}{l}
\left(\mathscr{E}_{0}\right)_{i, j}=\max \left(\mathscr{E}_{\text {inf }}, \min \left(1, \tilde{M}_{i, j}\right)\right), \\
p_{i, j}^{*, \theta}=\frac{\mathcal{S}_{L} p_{R}+\mathcal{S}_{R} p_{L}-\theta_{i, j} \mathcal{S}_{R} \mathcal{S}_{L}\left(\boldsymbol{u}_{R} \cdot \boldsymbol{n}_{i, j}-\boldsymbol{u}_{L} \cdot \boldsymbol{n}_{i, j}\right)}{\mathcal{S}_{R}+\mathcal{S}_{L}}, \\
u_{i, j}^{* \beta}=\frac{\mathcal{S}_{R} \boldsymbol{u}_{R} \cdot \boldsymbol{n}_{i, j}+\mathcal{S}_{L} \boldsymbol{u}_{L} \cdot \boldsymbol{n}_{i, j}-\beta_{i, j}\left(p_{R}-p_{L}\right)}{\mathcal{S}_{R}+\mathcal{S}_{L}} .
\end{array}\right.
$$

It can be noticed that the intermediate pressure and velocity formulas proposed in (29) are identical to the these written in (15) if and only if $\left(\theta_{i, j}, \beta_{i, j}\right)=(1,1)$. These coefficients can be assimilated to potential low-Mach corrections acting on the non-centered part of the momentum and the energy fluxes of the acoustic operator. Indeed, in the case of the acoustic contribution to the momentum flux, one can write

$$
p_{i, j}^{*, \theta}=p_{i, j}^{*}+\left(1-\theta_{i, j}\right) \frac{\mathcal{S}_{R} \mathcal{S}_{L}}{\mathcal{S}_{R}+\mathcal{S}_{L}}\left(\boldsymbol{u}_{R} \cdot \boldsymbol{n}_{i, j}-\boldsymbol{u}_{L} \cdot \boldsymbol{n}_{i, j}\right) .
$$

The second term is then similar to the $\theta$-correction of Dellacherie et al. [15] presented in Eq. (25).

In the sequel, by performing a non-dimensional Mach number expansion on the equalities given in the discrete acoustic kernel sub-space defined in (28), one tries to derive sufficient conditions on the scaling of the couple $\left(\theta_{i, j}, \beta_{i, j}\right)$ for which

$$
\mathcal{I}_{N} \subset\{\operatorname{Ker} \boldsymbol{P}\}_{N},
$$

is guaranteed. Inclusion (31) formally states that the discrete acoustic operator will not perturb initial discrete incompressible solutions after one time-step. For the sake of simplicity, let us introduce some notations:

$$
\begin{aligned}
\omega_{i, j}^{+} & =\frac{\mathcal{S}_{R}}{\mathcal{S}_{R}+\mathcal{S}_{L}}, \omega_{i, j}^{-}=\frac{\mathcal{S}_{L}}{\mathcal{S}_{R}+\mathcal{S}_{L}}, \\
\mathcal{D}_{i, j}^{p} & =\frac{\mathcal{S}_{R} \mathcal{S}_{L}}{\mathcal{S}_{R}+\mathcal{S}_{L}}, \mathcal{D}_{i, j}^{u}=\frac{1}{\mathcal{S}_{R}+\mathcal{S}_{L}} .
\end{aligned}
$$

The pressure $p^{*, \theta}$ and the velocity $u^{*, \beta}$ can thus be rewritten as

$$
\left\{\begin{array}{lll}
p^{*, \theta}=\frac{p_{R}+p_{L}}{2} & -\left(\omega_{i, j}^{+}-1 / 2\right)\left(p_{R}-p_{L}\right) & -\theta_{i, j} \mathcal{D}_{i, j}^{p}\left(\boldsymbol{u}_{R} \cdot \boldsymbol{n}_{i, j}-\boldsymbol{u}_{L} \cdot \boldsymbol{n}_{i, j}\right), \\
u^{*, \beta}=\frac{\boldsymbol{u}_{R} \cdot \boldsymbol{n}_{i, j}+\boldsymbol{u}_{L} \cdot \boldsymbol{n}_{i, j}}{2} & -\left(\omega_{i, j}^{-}-1 / 2\right)\left(\boldsymbol{u}_{R} \cdot \boldsymbol{n}_{i, j}-\boldsymbol{u}_{L} \cdot \boldsymbol{n}_{i, j}\right) & -\beta_{i, j} \mathcal{D}_{i, j}^{u}\left(p_{R}-p_{L}\right) .
\end{array}\right.
$$

In order to exhibit the scaling sufficient conditions contributing to inclusion (31), one first needs to derive the nondimensional version of $\{\operatorname{Ker} \boldsymbol{P}\}_{N}$, and decompose its constitutive relations in powers of the Mach number. Such a work is performed in the two following paragraphs in which the momentum and the energy discrete equalities are treated separately.

\subsubsection{Correction arising from the discrete momentum equation}

In the sequel, for the sake of simplicity, the non-dimensional variables are written the same way that the dimensional variables. In definition (28) of the discrete acoustic kernel, the non-dimensional equality related to the momentum equation with $\boldsymbol{U}_{L}=\boldsymbol{U}_{i}$ and $\boldsymbol{U}_{R}=\boldsymbol{U}_{j}$ writes:

$$
\sum_{j \in V(i)}\left(1-M^{2}\left(\mathscr{E}_{0}\right)_{i, j}^{2}\right)\left[\frac{1}{M^{2}}\left\{\frac{p_{j}+p_{i}}{2}-\left(\omega_{i, j}^{+}-1 / 2\right)\left(p_{j}-p_{i}\right)\right\}-\frac{\theta_{i, j}}{M} \mathcal{D}_{i, j}^{p}\left(\boldsymbol{u}_{j} \cdot \boldsymbol{n}_{i, j}-\boldsymbol{u}_{i} \cdot \boldsymbol{n}_{i, j}\right)\right] \boldsymbol{S}_{i, j}=\mathbf{0} .
$$

Recall that in equality (34), each non-dimensional term is a priori of order one and can be decomposed in Mach powers:

$$
\forall \phi \in\{\rho, \boldsymbol{u}, p\}, \phi=\phi^{(0)}+M \phi^{(1)}+M^{2} \phi^{(2)}+O\left(M^{3}\right) .
$$


Assuming that equality (34) implies that each contribution of the different Mach powers is null, one can write that for the $1 / M^{2}$ and the $1 / M$ terms:

$$
\begin{aligned}
& \sum_{j \in V(i)}\left\{\frac{p_{j}^{(0)}+p_{i}^{(0)}}{2}-\left(\left(\omega_{i, j}^{+}\right)^{(0)}-1 / 2\right)\left(p_{j}^{(0)}-p_{i}^{(0)}\right)\right\} \boldsymbol{S}_{i, j}=\mathbf{0}, \\
& \sum_{j \in V(i)}\left\{\frac{p_{j}^{(1)}+p_{i}^{(1)}}{2}-\left(\left(\omega_{i, j}^{+}\right)^{(0)}-1 / 2\right)\left(p_{j}^{(1)}-p_{i}^{(1)}\right)-\left(\omega_{i, j}^{+}\right)^{(1)}\left(p_{j}^{(0)}-p_{i}^{(0)}\right)-\theta_{i, j}^{(0)}\left(\mathcal{D}_{i, j}^{p}\right)^{(0)}\left(\boldsymbol{u}_{j}^{(0)} \cdot \boldsymbol{n}_{i, j}-\boldsymbol{u}_{i}^{(0)} \cdot \boldsymbol{n}_{i, j}\right)\right\} \boldsymbol{S}_{i, j}=\mathbf{0} .
\end{aligned}
$$

Hence, if one imposes $\forall(i, j)$

$$
\begin{aligned}
& \omega_{i, j}^{+}=\omega_{i, j}^{-}=\frac{1}{2}, \\
& \theta_{i, j}^{(0)}=0,
\end{aligned}
$$

a discrete solution belonging to $\mathcal{I}_{N}$ satisfies automatically relations (36). Let us underline that there is no need to impose condition (37a) if $\forall i$

$$
p_{i}=p^{(0),(1)}(t)+O\left(M^{2}\right)
$$

with $p^{(0),(1)}$ a time-dependent constant. However, along the calculation, there might exist discrete solutions fulfilling

$$
\sum_{j \in V(i)} \frac{p_{i}^{(0),(1)}+p_{j}^{(0),(1)}}{2} \boldsymbol{S}_{i, j}=\mathbf{0}
$$

without guaranteeing that $\forall(i, j), p_{i}^{(0),(1)}=p_{j}^{(0),(1)}$. These discrete solutions are called checkerboard modes and their appearance and amplification are studied in the framework of a linear wave equation in [32]. Condition (37a) then contributes to the checkerboard modes invariance (and thus their non-amplification) through the momentum equation of the discrete acoustic operator. Let us notice that condition (37a) holds by taking for $K=L, R$,

$$
\mathcal{S}_{K}=\mathcal{S}=\max \left(\rho_{L} c_{L}, \rho_{R} c_{R}\right) .
$$

Equality (40) corresponds exactly to the subcharacteristic condition proposed in [31,33] involved in the acoustic subsystem discretization of a Lagrange-Projection method. Condition (37b) is more classical and has been already exhibited by the same kind of calculations based on a two-dimensional Roe scheme in $[11,17]$.

In the following paragraph, equality (40) is fulfilled.

\subsubsection{Correction arising from the discrete energy equation}

Under equality (40), the intermediate pressure and velocity now simply write:

$$
\left\{\begin{aligned}
p^{*, \theta} & =\frac{p_{R}+p_{L}}{2} \\
u^{*, \beta} & =\frac{\boldsymbol{u}_{R} \cdot \boldsymbol{n}_{i, j}+\boldsymbol{u}_{L} \cdot \boldsymbol{n}_{i, j}}{2}-\beta_{i, j} \frac{\mathcal{S}\left(\boldsymbol{u}_{R} \cdot \boldsymbol{n}_{i, j}-\boldsymbol{u}_{L} \cdot \boldsymbol{n}_{i, j}\right)}{2}
\end{aligned}\right.
$$

Let us mention that, in the specific case where $\left(\theta_{i, j}, \beta_{i, j}\right)=(1,1)$, relations $(41)$ are identical to these obtained in $[31,33]$ in the context of Lagrange-Projection methods. In this section, attention is paid to the non-dimensional equality related to the energy equation in the definition of the discrete acoustic kernel (28). Such an equality reads:

$$
\sum_{j \in V(i)}\left(1-M^{2}\left(\mathscr{E}_{0}\right)_{i, j}^{2}\right)\left[\frac{p_{j}+p_{i}}{2}-M \theta_{i, j} \frac{\mathcal{S}_{i, j}}{2}\left(\boldsymbol{u}_{j} \cdot \boldsymbol{n}_{i, j}-\boldsymbol{u}_{i} \cdot \boldsymbol{n}_{i, j}\right)\right] \times\left[\frac{\boldsymbol{u}_{j} \cdot \boldsymbol{n}_{i, j}+\boldsymbol{u}_{i} \cdot \boldsymbol{n}_{i, j}}{2}-\frac{\beta_{i, j}}{M} \frac{1}{2 \mathcal{S}_{i, j}}\left(p_{j}-p_{i}\right)\right]\left|S_{i, j}\right|=0 .
$$


After having decomposed equality (42) in powers of the Mach number, one obtains:

Order $1 / M$ :

$$
\sum_{j \in V(i)}\left[\frac{p_{j}^{(0)}+p_{i}^{(0)}}{2}\right] \times\left[\beta_{i, j}^{(0)} \frac{1}{2 \mathcal{S}_{i, j}^{(0)}}\left(p_{j}^{(0)}-p_{i}^{(0)}\right)\right]\left|S_{i, j}\right|=0
$$

Order 1:

$$
\begin{aligned}
& \sum_{j \in V(i)}\left[\frac{p_{j}^{(1)}+p_{i}^{(1)}}{2}-\theta_{i, j}^{(0)} \frac{\mathcal{S}_{i, j}^{(0)}}{2}\left(\boldsymbol{u}_{j}^{(0)} \cdot \boldsymbol{n}_{i, j}-\boldsymbol{u}_{i}^{(0)} \cdot \boldsymbol{n}_{i, j}\right)\right] \times\left[-\beta_{i, j}^{(0)} \frac{1}{2 \mathcal{S}_{i, j}^{(0)}}\left(p_{j}^{(0)}-p_{i}^{(0)}\right)\right]\left|S_{i, j}\right| \\
+ & \sum_{j \in V(i)}\left[\frac{p_{j}^{(0)}+p_{i}^{(0)}}{2}\right] \times\left[\frac{\boldsymbol{u}_{j}^{(0)} \cdot \boldsymbol{n}_{i, j}+\boldsymbol{u}_{i}^{(0)} \cdot \boldsymbol{n}_{i, j}}{2}-\left(\beta_{i, j} \frac{1}{2 \mathcal{S}_{i, j}}\left(p_{j}-p_{i}\right)\right)^{(1)}\right]\left|S_{i, j}\right|=0 .
\end{aligned}
$$

$\underline{\text { Order } M \text { : }}$

$$
\begin{aligned}
& \sum_{j \in V(i)}-\left(\mathscr{E}_{0}\right)_{i, j}^{2}\left[\frac{p_{j}^{(0)}+p_{i}^{(0)}}{2}\right] \times\left[-\beta_{i, j}^{(0)} \frac{1}{2 \mathcal{S}_{i, j}^{(0)}}\left(p_{j}^{(0)}-p_{i}^{(0)}\right)\right]\left|S_{i, j}\right| \\
+ & \sum_{j \in V(i)}\left[\frac{p_{j}^{(2)}+p_{i}^{(2)}}{2}-\left(\theta_{i, j} \frac{\mathcal{S}_{i, j}}{2}\left(\boldsymbol{u}_{j} \cdot \boldsymbol{n}_{i, j}-\boldsymbol{u}_{i} \cdot \boldsymbol{n}_{i, j}\right)\right)^{(1)}\right] \times\left[-\beta_{i, j}^{(0)} \frac{1}{2 \mathcal{S}_{i, j}^{(0)}}\left(p_{j}^{(0)}-p_{i}^{(0)}\right)\right]\left|S_{i, j}\right| \\
+ & \sum_{j \in V(i)}\left[\frac{p_{j}^{(1)}+p_{i}^{(1)}}{2}-\theta_{i, j}^{(0)} \frac{\mathcal{S}_{i, j}^{(0)}}{2}\left(\boldsymbol{u}_{j}^{(0)} \cdot \boldsymbol{n}_{i, j}-\boldsymbol{u}_{i}^{(0)} \cdot \boldsymbol{n}_{i, j}\right)\right] \times\left[\frac{\boldsymbol{u}_{j}^{(0)} \cdot \boldsymbol{n}_{i, j}+\boldsymbol{u}_{i}^{(0)} \cdot \boldsymbol{n}_{i, j}}{2}-\left(\beta_{i, j} \frac{1}{2 \mathcal{S}_{i, j}}\left(p_{j}-p_{i}\right)\right)^{(1)}\right]\left|S_{i, j}\right| \\
+ & \sum_{j \in V(i)}\left[\frac{p_{j}^{(0)}+p_{i}^{(0)}}{2}\right] \times\left[\frac{\boldsymbol{u}_{j}^{(1)} \cdot \boldsymbol{n}_{i, j}+\boldsymbol{u}_{i}^{(1)} \cdot \boldsymbol{n}_{i, j}}{2}-\left(\beta_{i, j} \frac{1}{2 \mathcal{S}_{i, j}}\left(p_{j}-p_{i}\right)\right)^{(2)}\right]\left|S_{i, j}\right|=0 .
\end{aligned}
$$

Consider a discrete solution that belongs to $\mathcal{I}_{N}$. Assume additionally that the pressure decomposition (38) holds. As a consequence one can observe that equality (43) is automatically fulfilled. Besides, equality (44) becomes:

$$
p^{(0),(1)}(t) \sum_{j \in V(i)} \frac{\boldsymbol{u}_{j}^{(0)}+\boldsymbol{u}_{i}^{(0)}}{2} \cdot \boldsymbol{S}_{i, j}=0 .
$$

Equality (46) holds automatically as it corresponds exactly to the discrete divergence-free constraint which partly defines $\mathcal{I}_{N}$. Note that, if the fluid is endowed with an ideal gas equation of state (EOS) such that

$$
\rho \varepsilon=\frac{p}{\gamma-1}, \rho c^{2}=\gamma p
$$

with $\gamma$ the specific heat ratio, the discrete terms

$$
\sum_{j \in V(i)}\left[\frac{p_{j}^{(0)}+p_{i}^{(0)}}{2}\right] \times\left[\frac{\boldsymbol{u}_{j}^{(0)} \cdot \boldsymbol{n}_{i, j}+\boldsymbol{u}_{i}^{(0)} \cdot \boldsymbol{n}_{i, j}}{2}\right]\left|S_{i, j}\right|,
$$

in equality (44), are consistent (up the $\gamma$ factor) with

$$
\left(\rho c^{2}\right)^{(0)} \nabla \cdot \boldsymbol{u}^{(0)}
$$

which intervenes in the zeroth-order continuous asymptotic pressure equation related to the Euler system:

$$
\partial_{t} p^{(0)}+\boldsymbol{u}^{(0)} \cdot \nabla p^{(0)}+\left(\rho c^{2}\right)^{(0)} \nabla \cdot \boldsymbol{u}^{(0)}=0 .
$$


Besides, the most intricate equality (45) simplifies as:

$$
\begin{aligned}
& \sum_{j \in V(i)}\left[p^{(0),(1)}(t)-\theta_{i, j}^{(0)} \frac{\mathcal{S}_{i, j}^{(0)}}{2}\left(\boldsymbol{u}_{j}^{(0)} \cdot \boldsymbol{n}_{i, j}-\boldsymbol{u}_{i}^{(0)} \cdot \boldsymbol{n}_{i, j}\right)\right] \times\left[\frac{\boldsymbol{u}_{j}^{(0)} \cdot \boldsymbol{n}_{i, j}+\boldsymbol{u}_{i}^{(0)} \cdot \boldsymbol{n}_{i, j}}{2}\right]\left|S_{i, j}\right| \\
+ & \sum_{j \in V(i)}\left[p^{(0),(1)}(t)\right] \times\left[\frac{\boldsymbol{u}_{j}^{(1)} \cdot \boldsymbol{n}_{i, j}+\boldsymbol{u}_{i}^{(1)} \cdot \boldsymbol{n}_{i, j}}{2}-\beta_{i, j}^{(0)} \frac{1}{2 \mathcal{S}_{i, j}^{(0)}}\left(p_{j}^{(2)}-p_{i}^{(2)}\right)\right]\left|S_{i, j}\right|=0 .
\end{aligned}
$$

Using the discrete divergence-free relation, equality (46) reduces to

$$
\begin{aligned}
\sum_{j \in V(i)}-\theta_{i, j}^{(0)} \frac{\mathcal{S}_{i, j}^{(0)}}{2}\left(\boldsymbol{u}_{j}^{(0)} \cdot \boldsymbol{n}_{i, j}-\boldsymbol{u}_{i}^{(0)} \cdot \boldsymbol{n}_{i, j}\right) \times\left[\frac{\boldsymbol{u}_{j}^{(0)}+\boldsymbol{u}_{i}^{(0)}}{2}\right] \cdot \boldsymbol{S}_{i, j} \\
-p^{(0),(1)}(t) \sum_{j \in V(i)} \beta_{i, j}^{(0)} \frac{1}{2 \mathcal{S}_{i, j}^{(0)}}\left(p_{j}^{(2)}-p_{i}^{(2)}\right)\left|S_{i, j}\right|=0 .
\end{aligned}
$$

One can observe that the remaining terms in equality (52) appear only because of the diffusive part of the intermediate pressure and velocity definition (41). As a consequence, by imposing that $\forall(i, j)$

$$
\begin{aligned}
& \theta_{i, j}^{(0)}=0, \\
& \beta_{i, j}^{(0)}=0,
\end{aligned}
$$

one ensures that equality (52) holds. The above formal analysis suggests that, in the context of stationary discrete solutions, low-Mach corrections applied to the non-centered part of the intermediate pressure and velocity of the energy flux ensure that, up to the order $O\left(M^{2}\right)$, the discrete acoustic operator does not perturb discrete incompressible solutions. Conversely, if $\left(\theta_{i, j}^{(0)}, \beta_{i, j}^{(0)}\right) \neq(0,0)$, equality (52) does not necessarily hold.

In the case of a fluid endowed with an ideal gas EOS, the non-dimensional energy equation can be formally read, after having discarded the kinetic energy terms scaling as $O\left(M^{2}\right)$, as a non-dimensional pressure equation. Then, in the context of stationary flows, the energy flux contributions associated with equality (52) might distort an incompressible discrete solution by creating a non-dimensional pressure of order $O(M)$. Let us mention that in the references $[11,17]$ that focus on the same topic, no low-Mach correction is applied to the non-dimensional energy flux of the Godunov-like schemes at stake. Indeed, in their approach, the formal expansion in Mach powers related to the energy PDE is only carried out up to terms scaling as $O(1)$. The authors notably ensure that, for ideal EOS, the appropriate zeroth-order incompressible pressure equation is retrieved at discrete level. However, nothing is told about the possibility, for their scheme, to transfer energy flux contributions scaling as $O(M)$ into the non-dimensional pressure variable from one time-step to another.

Finally, the proposed numerical flux at interface $S_{i, j}$ reads:

$$
\boldsymbol{\Phi}_{i, j}^{\mathrm{FVS}}=\boldsymbol{\Gamma}_{i, j}+\left(1-\left(\mathscr{E}_{0}\right)_{i, j}^{2}\right) \boldsymbol{\Pi}_{i, j}
$$

with for $K=L, R$,

$$
\left\{\begin{array}{l}
\boldsymbol{\Gamma}_{i, j}=\frac{1}{2}\left[u^{*} \boldsymbol{W}_{R}+u^{*} \boldsymbol{W}_{L}-\left|u^{*}\right|\left(\boldsymbol{W}_{R}-\boldsymbol{W}_{L}\right)\right]+\left(\mathscr{E}_{0}\right)_{i, j}^{2}\left(\begin{array}{c}
0 \\
p^{*, \theta} \boldsymbol{n}_{i, j} \\
0
\end{array}\right), \quad \boldsymbol{W}_{K}=\left(\begin{array}{c}
\rho_{K} \\
(\rho \boldsymbol{u})_{K} \\
(\rho e)_{K}+\mathscr{E}_{0}^{2} p_{K}
\end{array}\right), \\
\boldsymbol{\Pi}_{i, j}=\left(\begin{array}{c}
0 \\
p^{*, \theta} \boldsymbol{n}_{i, j} \\
p^{*, \theta} u^{*}
\end{array}\right),
\end{array}\right.
$$

and,

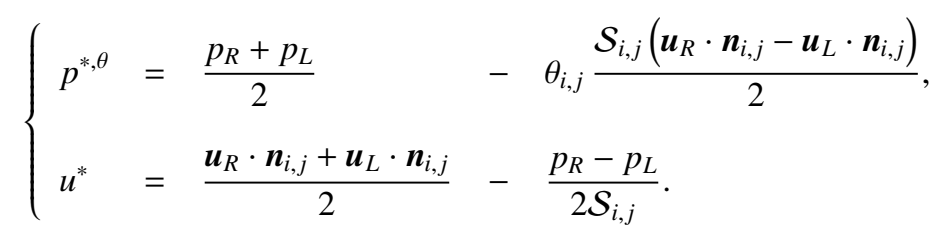


Besides, $\mathcal{S}_{i, j}=\max \left(\rho_{R} c_{R}, \rho_{L} c_{L}\right), \theta_{i, j}=\tilde{M}_{i, j}$ with $\tilde{M}_{i, j}$ the local Mach number defined in Eq. (23) for a twodimensional flow. The splitting parameter is set to be $\left(\mathscr{E}_{0}\right)_{i, j}=\max \left(\mathscr{E}_{\text {inf }}, \min \left(1, \tilde{M}_{i, j}\right)\right)$ with $\mathscr{E}_{\text {inf }}=10^{-8}$. Although the above formal low-Mach number analysis compels to apply a low-Mach correction to the non-centered part of the intermediate velocity $u^{*}$ in the energy flux, the numerical anti-diffusion that it brings endangers the stability of the proposed flux vector splitting approach. As a consequence, it has been removed from the present numerical scheme.

All of the computations presented in the following have been performed with the fast transient dynamics software for fluids and structures Europlexus [34].

In the sequel, numerical test cases are provided in order to assess the accuracy of the proposed flux vector splitting scheme.

\section{Numerical tests}

The numerical results presented below go through a wide variety of flow regimes depending on the maximal value of the Mach number. The first test-case is the one-dimensional Sod's shock tube [35]. It aims at testing the capacity of the present scheme to capture fast compressible shock and rarefaction waves in regions where the Mach number is close to one. Then, another Riemann problem whose analytical solution is a stationary contact discontinuity. Here, one checks the ability of the proposed flux vector splitting scheme to exactly capture, on a large convective time-scale, a solution of the compressible Euler system associated with the slow material-wave dynamics.

Subsequently, one is interested in approximating, with a sufficient accuracy, stationary solutions related to the two-dimensional incompressible Euler system. In that case, the physical time of simulation is systematically based on the material velocity and the characteristic length-scale of the non-dimensional cartesian mesh is larger than the Mach number. In this context, the capacity of the present scheme to tackle the low Mach number accuracy issue is assessed. The first 2-D test-case is a stationary Gresho vortex [36]. The second one is a low Mach number flow in a channel with a bump [14].

Finally, a case composed of a fast acoustic wave, associated with a Mach number of order one, crossing a stationary Gresho vortex is studied. Here, the capacity of the proposed splitting to adapt locally according to the variations of the Mach number so that to either capture compressible waves or preserve stationary incompressible solutions is at stake.

Three numerical schemes are here compared: the classical HLLC scheme (given in Eq. (5)), the modified HLLC scheme labelled "HLLC-Corr" in the following (see Eq. (25)) and the present flux-vector splitting (detailed in Eq. (54)).

Let us end this test-case presentation by saying that in every simulation, the discrete time-step $\Delta t^{n}$ is built such that the Courant number $C$ based on the Euler eigenvalues in Eq. (4) is equal to 0.45.

\subsection{Test 1: Sod's shock tube}

The initial conditions related to this one-dimensional Riemann problem are displayed in Table 1. The solution is

\begin{tabular}{|c|c|}
\hline Left state & Right state \\
\hline$\rho_{L}^{0}=1 \mathrm{~kg} . \mathrm{m}^{-3}$ & $\rho_{R}^{0}=0.125 \mathrm{~kg} \cdot \mathrm{m}^{-3}$ \\
\hline$p_{L}^{0}=1 \mathrm{bar}$ & $p_{R}^{0}=0.1 \mathrm{bar}$ \\
\hline$u_{L}^{0}=0 \mathrm{~m} . \mathrm{s}^{-1}$ & $u_{R}^{0}=0 \mathrm{~m} . \mathrm{s}^{-1}$ \\
\hline
\end{tabular}

Table 1: Sod's shock tube: initial conditions

made of a left-going rarefaction wave, a right-going contact discontinuity and a right-going shock wave. The maximal Mach number, reached in the tail of the rarefaction wave is $M \approx 0.92$. The time of the simulation, based on the shock wave speed, is $T_{\text {end }}=4.5 \times 10^{-4} \mathrm{~s}$. In Figure 3, the pressure, velocity and density profiles of the computed solutions for the three mentioned schemes are plotted. The mesh is made of $10^{3}$ cells. It turns out that the proposed FVS scheme manages to capture the fast compressible rarefaction and shock waves. 


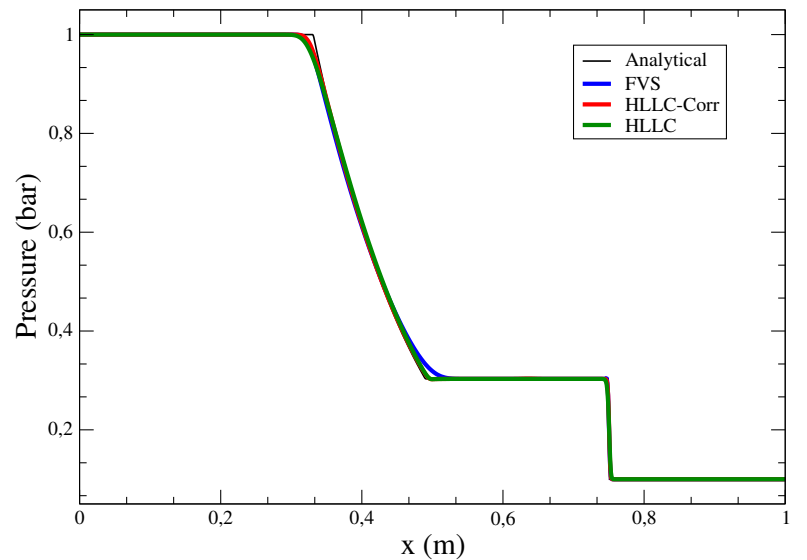

(a) $p$ (bar)

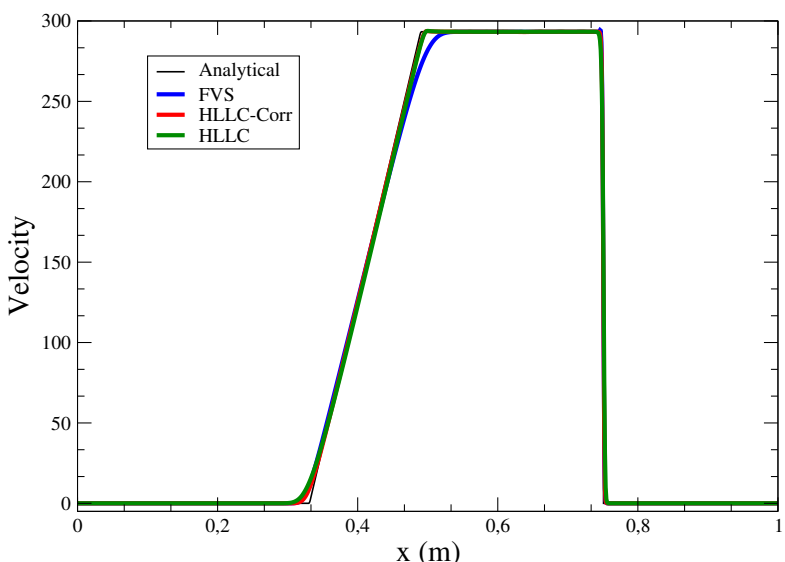

(b) $u\left(\mathrm{~m} \cdot \mathrm{s}^{-1}\right)$

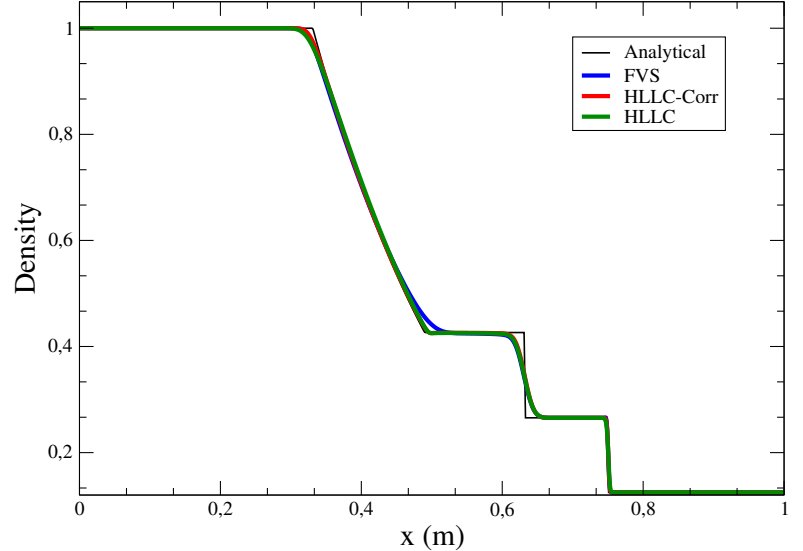

(c) $\rho\left(\mathrm{kg} \cdot \mathrm{m}^{-3}\right)$

Figure 3: Profiles of (a) the pressure, (b) the velocity and (c) the density for the simulation of the Sod's shock tube: mesh made of $10^{3}$ cells, Courant number $C=0.45$; comparison between the analytical and the computed solutions. 
The profiles obtained are equivalent. The proposed FVS scheme is slightly more diffusive at the tail of the rarefaction wave compared to the other HLLC schemes. Indeed, in this region, the Mach number is almost equal to one and the pressure part, $\left(1-\mathscr{E}_{0}^{2}\right) \boldsymbol{\Pi}_{i, j}$, of the present splitting cancels out. The resulting scheme is then based on a simple upwinding that, contrary to the two HLLC-type schemes, is not designed to approximate the fastest waves appearing in the flow.

\subsection{Test 2: Stationary contact discontinuity}

In this test-case, initial conditions written in Table 2 are such that pressure is constant and velocity is null in the overall computational domain. Hence, the analytical solution is made of a single stationary contact wave. The time of the simulation is $T_{\text {end }}=10^{-1} \mathrm{~s}$. Similarly to the previous case, pressure, velocity and density profiles at final time

\begin{tabular}{|c|c|}
\hline Left state & Right state \\
\hline$\rho_{L}^{0}=10 \mathrm{~kg} \cdot \mathrm{m}^{-3}$ & $\rho_{R}^{0}=1 \mathrm{~kg} \cdot \mathrm{m}^{-3}$ \\
\hline$p_{L}^{0}=1 \mathrm{bar}$ & $p_{R}^{0}=1 \mathrm{bar}$ \\
\hline$u_{L}^{0}=0 \mathrm{~m} . \mathrm{s}^{-1}$ & $u_{R}^{0}=0 \mathrm{~m} . \mathrm{s}^{-1}$ \\
\hline
\end{tabular}

Table 2: Stationary contact wave: initial conditions

are displayed in Figure 4, for a $10^{3}$ cells mesh. It turns out that the proposed FVS approach is able to preserve exactly the constant pressure and velocity profile as well as the initial stationary density jump. Similarly to [9], this property holds because of the pressure flux discretization proposed in Eq. (56). Indeed, it relies on an acoustic approximate Riemann solver such that the non-centered part of the produced intermediate velocity (respectively the intermediate pressure) is a function of the pressure difference (respecitvely of the velocity difference). Let us mention that in this test-case, the parameter $\mathscr{E}_{0}$ is equal to its inferior bound $\mathscr{E}_{\text {inf }}=10^{-8}$ because of the zero-velocity field. The splitting thus decouples convection at material velocity from acoustic wave production.

\subsection{Test 3: 2-D stationary vortex}

The third test-case is a stationary version of the vortex proposed by Gresho and Chan [36, 37] and also considered in [17]. At the initial time $t=0$, a vortex of radius $R=0.4 \mathrm{~m}$ whose the center is located at the position $\left(x_{0}, y_{0}\right)=(1,1)$ is prescribed:

$$
\begin{aligned}
& \left\{\begin{aligned}
\rho & =\rho_{0}, \\
u & =-\frac{y-y_{0}}{r} \delta u, \\
v & =\frac{x-x_{0}}{r} \delta u, \\
p & =p_{0}+\delta p,
\end{aligned}\right. \\
& \text { with } \delta u=M_{0} c_{0} \begin{cases}2 r / R & \text { if } 0 \leq r<R / 2, \\
2(1-r / R) & \text { if } R / 2 \leq r<R, \\
0 & \text { if } R \leq r,\end{cases} \\
& \text { and } \delta p=p_{0} M_{0}^{2} \begin{cases}2(r / R)^{2} & \text { if } 0 \leq r \leq R / 2 \\
2(r / R)^{2}+4(1-2 r / R+\log (2 r / R)) & \text { if } R / 2<r \leq R \\
-2+4 \log (2) & \text { if } R<r\end{cases}
\end{aligned}
$$

where $\rho_{0}=1 \mathrm{~kg} \cdot \mathrm{m}^{-3}, p_{0}=1$ bar, $M_{0}=10^{-2}$ with $c_{0}=\sqrt{\gamma p_{0} / \rho_{0}}$ and $r=\sqrt{\left(x-x_{0}\right)^{2}+\left(y-y_{0}\right)^{2}}$. Let us underline that the velocity and pressure fields are smooth solutions of the stationary incompressible Euler system:

$$
\left\{\begin{array}{lll}
\nabla \cdot \boldsymbol{u} & =0 \\
\rho_{0} \boldsymbol{u} \cdot \nabla \boldsymbol{u}+\nabla p & =\mathbf{0}
\end{array}\right.
$$




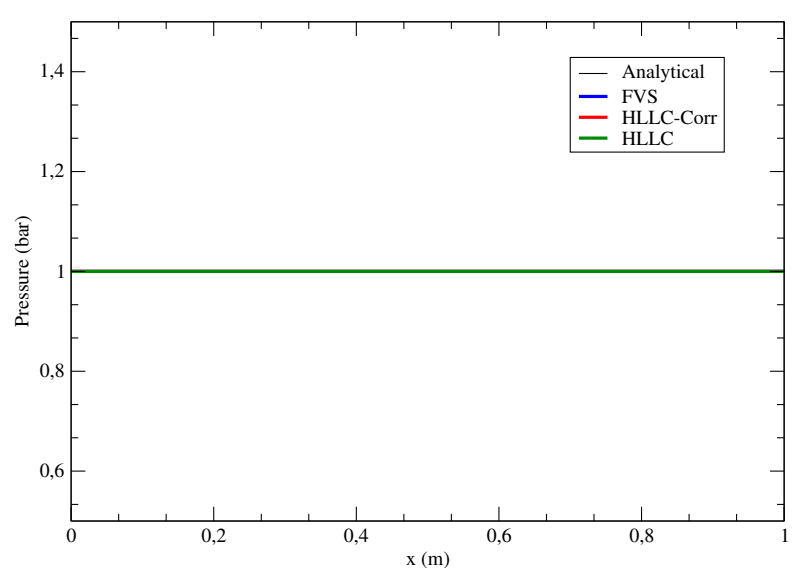

(a) $p$ (bar)

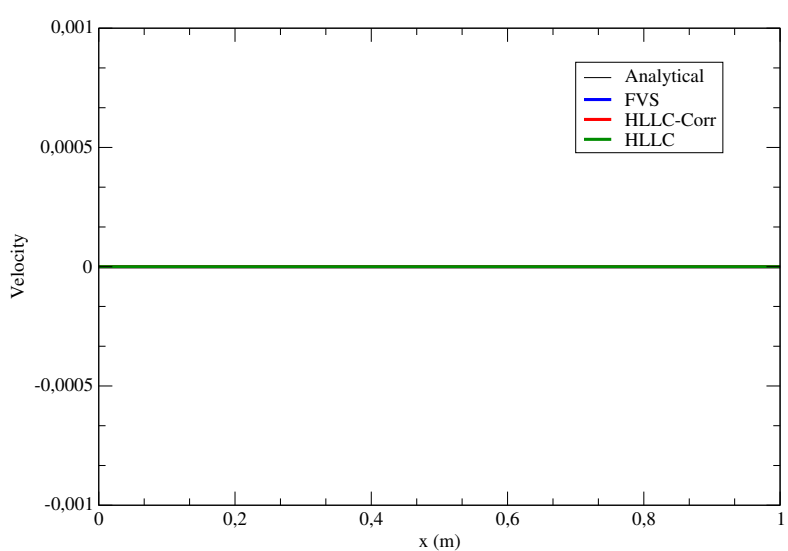

(b) $u\left(\mathrm{~m} \cdot \mathrm{s}^{-1}\right)$

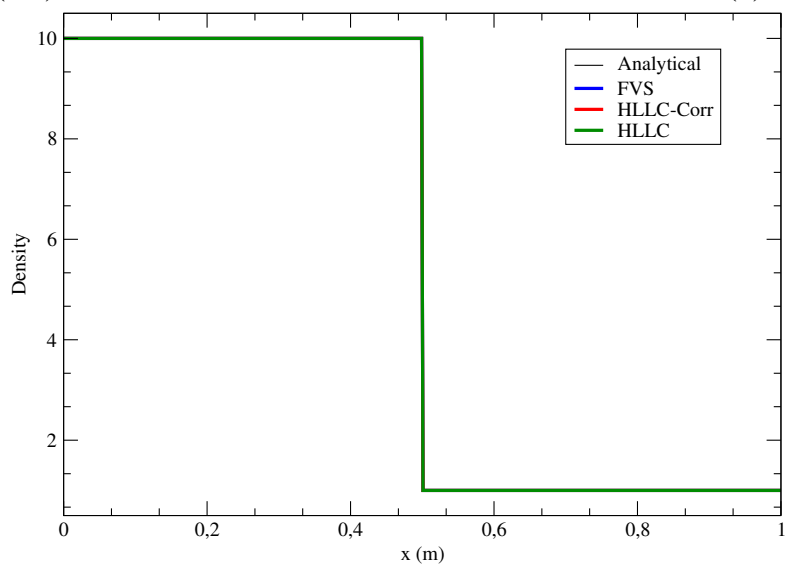

(c) $\rho\left(\mathrm{kg} \cdot \mathrm{m}^{-3}\right)$

Figure 4: Profiles of (a) the pressure, (b) the velocity and (c) the density for the simulation of the stationary contact wave: mesh made of $10^{3}$ cells, Courant number $C=0.45$; comparison between the analytical and the computed solutions. 
The 2-D computation domain corresponds to $[0, L] \times[0, L]$ with $L=2 \mathrm{~m}$. All the computations are done with $50 \times 50$ cells. The final time of the computation is $t=0.5 \mathrm{~s}$ corresponding approximately to the convective time scale $L / u_{0}$ with $u_{0}=M_{0} c_{0}$. Transmissive boundary conditions are used for the inlet, outlet, top and bottom boundaries.

The numerical solutions obtained with the HLLC scheme, the modified HLLC solver and the present FVS approach are compared in Figure 5 and Figure 6. The profiles of the Mach number, the velocity norm $|\boldsymbol{u}|$ as well as the non-dimensional incompressible pressure component $\left(p-p_{0}\right) / p_{0}$ are displayed. The numerical results obtained

(a) HLLC scheme

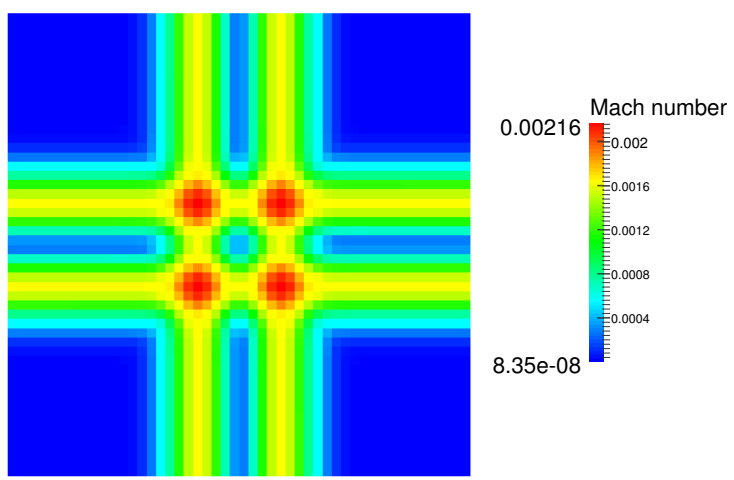

(b) HLLC-Corr scheme

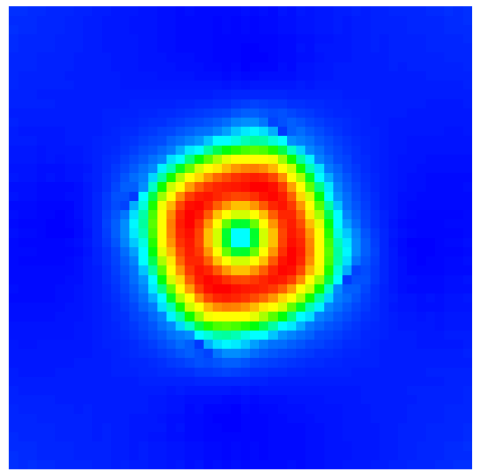

(c) present FVS scheme

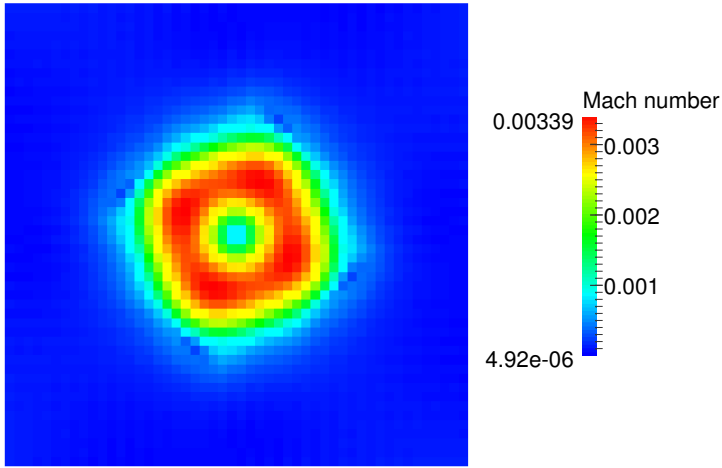

Figure 5: Mach number for simulations of the stationary vortex: $M_{0}=10^{-2}$, mesh made of $50 \times 50$ cells, Courant number $C=0.45$; with (a) the HLLC scheme, (b) the modified HLLC scheme and (c) the present approach.

with the HLLC solver deteriorate the vortex leading to non-cylindrical structures as observed in Figure 5-(a) on the Mach number profile. In contrast, the present FVS approach gives similar results as the modified HLLC scheme with a strongly improved resolution of the vortex.

This is also observed on the velocity norm and non-dimensional incompressible pressure profiles plotted on Figure 6. Let us finally underline that, for both HLLC-Corr and the present FVS approach, structures looking like checker board modes seem to appear on the profile of $\left(p-p_{0}\right) / p_{0}$. A close look on the scale of these modes reveals that their amplitude is larger in the case of the HLLC-Corr scheme than in the proposed method. 
(a) HLLC-Corr scheme

(1)
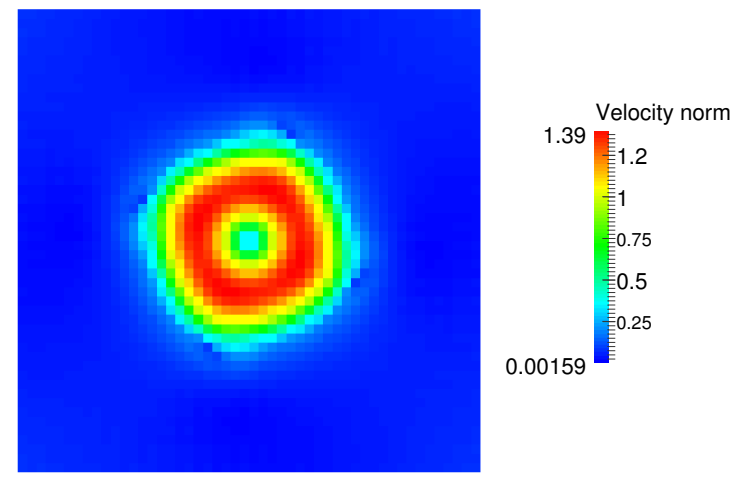

(2)

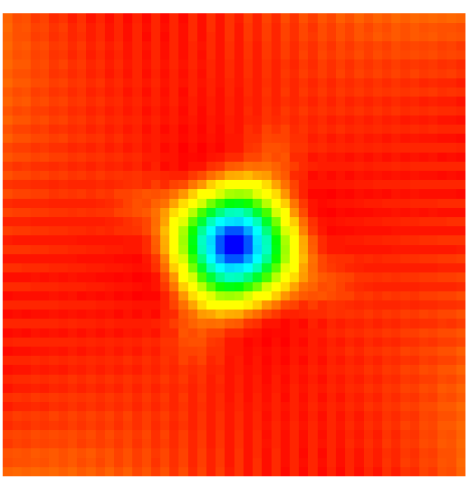

(b) present FVS scheme
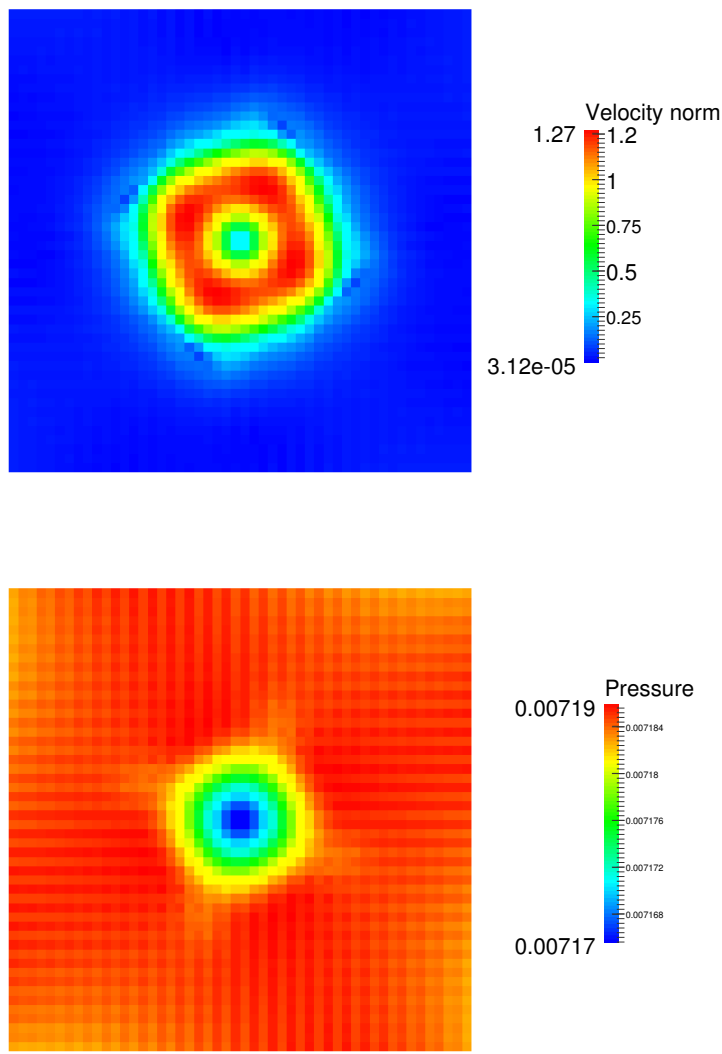

Figure 6: Profiles of (1) the velocity norm $|\boldsymbol{u}|$, (2) $\left(p-p_{0}\right) / p_{0}$ for simulations of the stationary vortex: $M_{0}=10^{-2}$, mesh made of $50 \times 50$ cells, Courant number $C=0.45$; with (a) the HLLC scheme, (b) the modified HLLC scheme and (c) the present approach. 


\subsection{Test 4: Subsonic flow in a channel with a bump}

The fourth test-case considered here consists of a low-velocity compressible flow in a channel with a bump as proposed in [14]. The initial flow conditions are characterized by:

$$
\rho_{0}=1.2 \mathrm{~kg} \cdot \mathrm{m}^{-3}, \quad p_{0}=1 \mathrm{bar} \text { and } u_{0}=M_{0} c_{0},
$$

with $c_{0}=\sqrt{\gamma p_{0} / \rho_{0}}$. The 2-D computation domain corresponds to $[-2 L, 2 L] \times[0, L]$ with $L=1 \mathrm{~m}$. The numerical solutions obtained with the classical HLLC solver, the modified HLLC scheme and the present approach are compared. Two set of computations are considered in the following: the first with $M_{0}=10^{-2}$ and the second with $M_{0}=10^{-3}$. All the computations are done with $64 \times 20$ cells and the Courant number $C=0.45$. For the case $M_{0}=10^{-2}$, the final time of the computation is $t=1.17 \mathrm{~s}$ corresponding approximately to the convective time scale $4 L / u_{0}$. It is ten times longer in the case $M_{0}=10^{-3}$. Inlet and outlet boundary conditions are obtained using the corresponding Riemann invariants whereas upper and lower boundaries are set to be walls.

The numerical results are plotted in Figure 7 and Figure 8. The Mach number, the non-dimensional incompressible pressure $\left(p-p_{0}\right) / p_{0}$ as well as the density profiles are displayed. First of all, one can observe that the numerical solution given by the classical HLLC scheme is not correct as observed in the Mach number field. Indeed, as already proved in [12], the stationary solution captured by the upwind solver corresponds to a balance of acoustic waves instead of an approximation of the incompressible field for which the Bernouilli's principle must hold. The numerical density fields obtained with the HLLC scheme in conjunction with the classical low-Mach correction on the momentum equation is not correct too. In contrast, the present approach using a correction on both momentum and energy equation gives satisfactory numerical results on all the variables.

In the case $M_{0}=10^{-3}$, the profiles of the Mach number, of $\left(p-p_{0}\right) / p_{0}$ and of the density are shown in Figure 9. The present FVS approach is only compared to the HLLC-Corr scheme.

The quality of the profiles between both schemes is similar for the Mach number and the non-dimensional incompressible pressure variables. Besides, as already observed previously, a low-Mach number correction holding on the non-centered part of $p^{*}$ in the energy flux allows to retrieve an appropriate profile for the density. 
(a) HLLC scheme
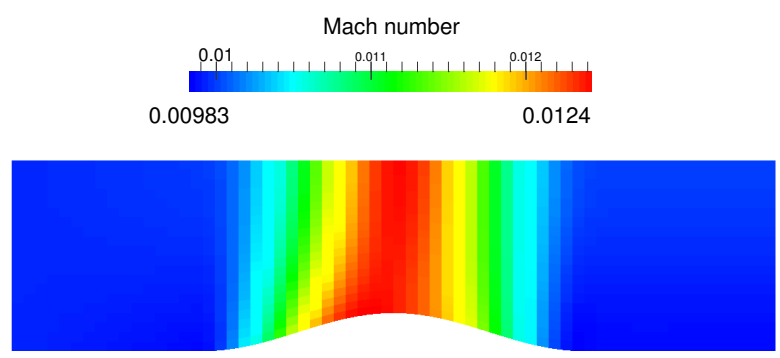

(b) HLLC-Corr scheme

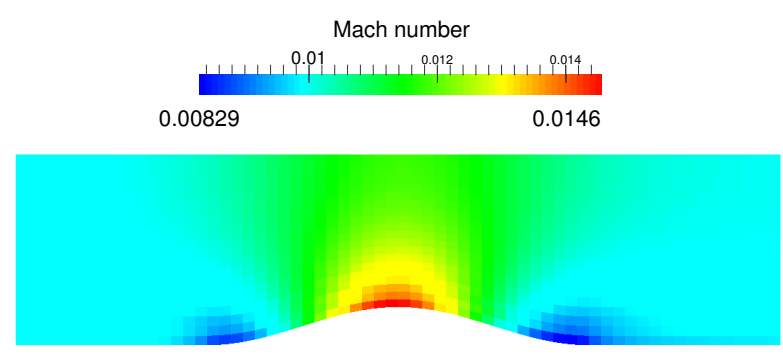

(c) present FVS scheme

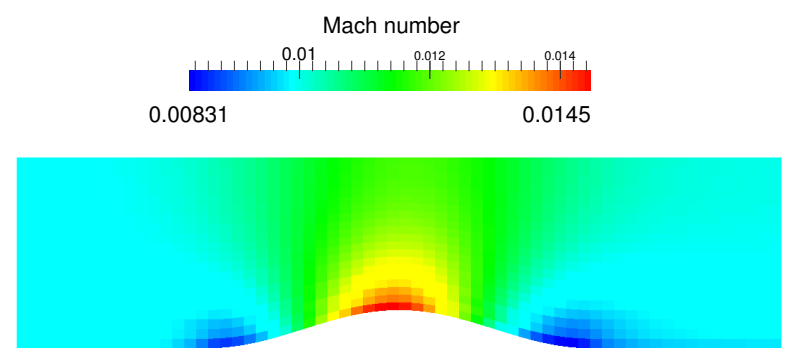

Figure 7: Mach number for simulations of the subsonic flow in a channel with a bump: $M_{0}=10^{-2}$, mesh made of $64 \times 20$ cells, Courant number $C=0.45$; with (a) the HLLC scheme, (b) the modified HLLC scheme and (c) the present approach. 


\section{(a) HLLC-Corr scheme}

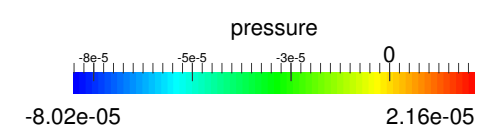

(1)

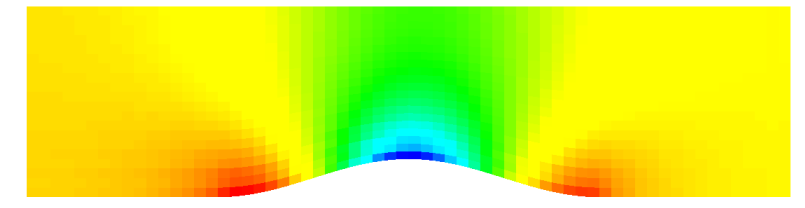

(2)
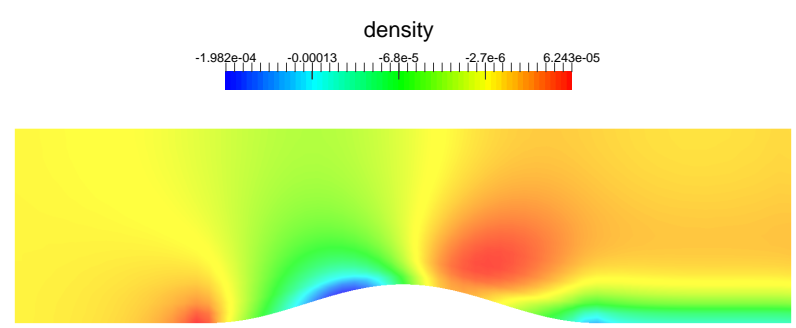

(b) present FVS scheme
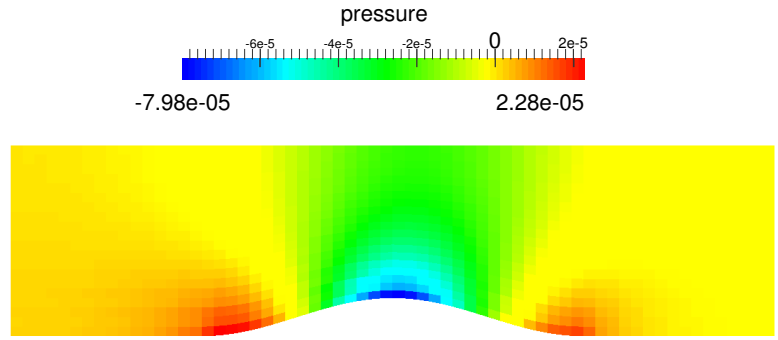

density

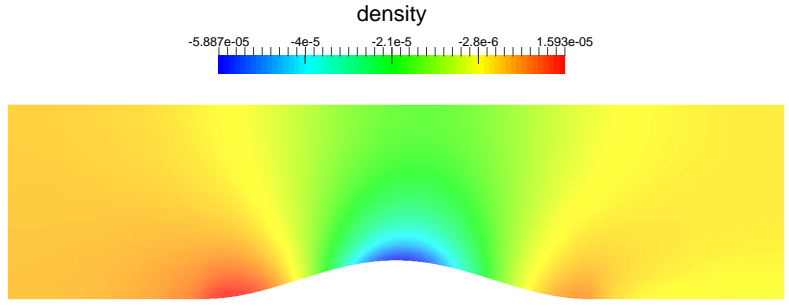

Figure 8: Profiles of (1) $\left(p-p_{0}\right) / p_{0}$ and (2) $\left(\rho-\rho_{0}\right) / \rho_{0}$ for simulations of the subsonic flow in a channel with a bump: $M_{0}=10^{-2}$, mesh made of $64 \times 20$ cells, Courant number $C=0.45$; with (a) the HLLC scheme, (b) the modified HLLC scheme and (c) the present approach. 
(a) HLLC-Corr scheme

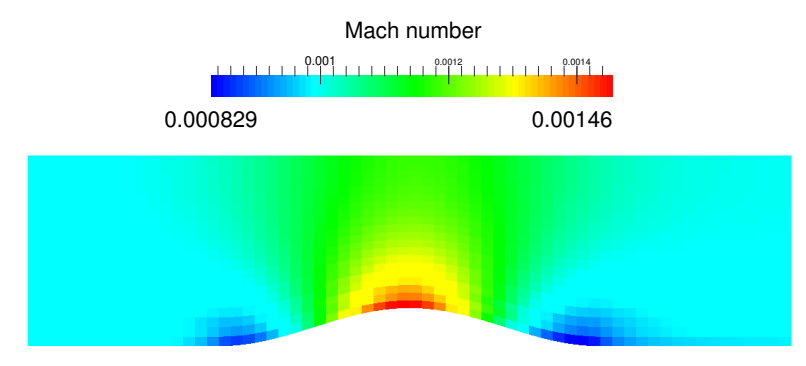

(1)

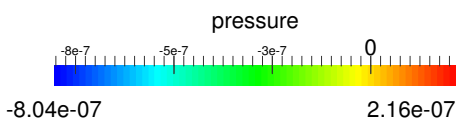

(2)

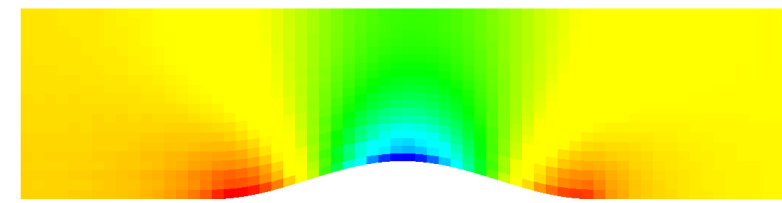

density

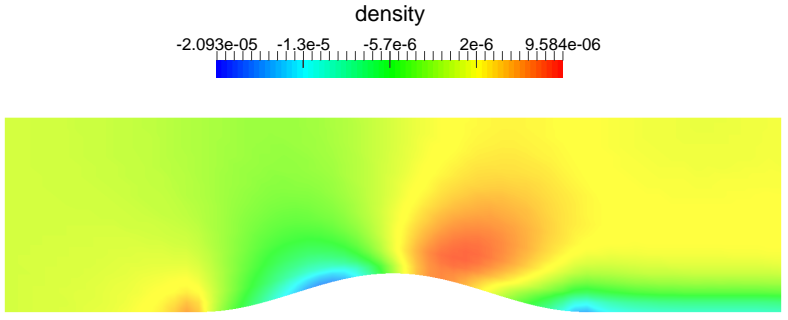

(3) (b) present FVS scheme

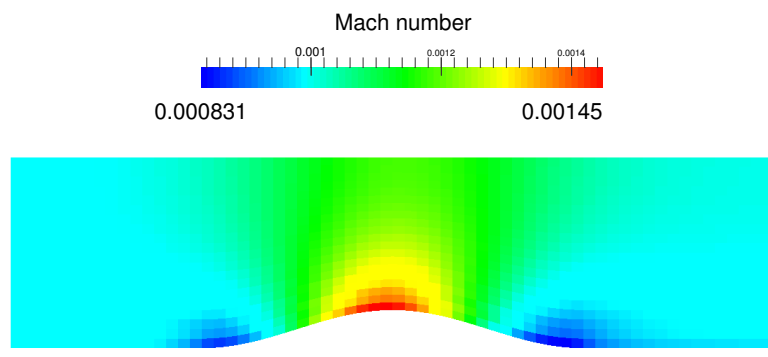

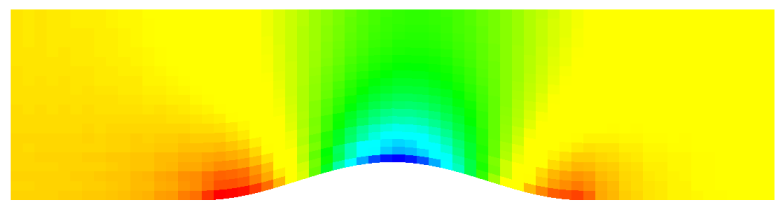

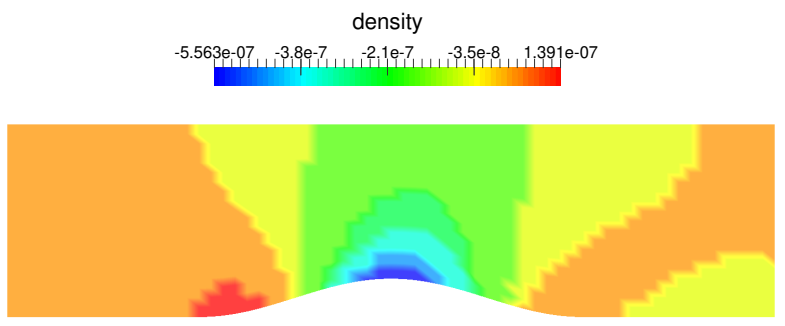

Figure 9: Profiles of (1) the Mach number, (2) $\left(p-p_{0}\right) / p_{0}$ and (3) $\left(\rho-\rho_{0}\right) / \rho_{0}$ for simulations of the subsonic flow in a channel with a bump: $M_{0}=10^{-3}$, mesh made of $64 \times 20$ cells, Courant number $C=0.45$; with (a) the modified HLLC scheme, (b) the present approach. 


\subsection{Test 5: Interaction acoustic wave with a 2-D stationary vortex}

The last test-case has been insipred from the work of Miczek and co-authors [38]. The computational domain represents a pipe section whose diameter is $L=2 \mathrm{~m}$ and length is $5 \times L=10 \mathrm{~m}$. The 2-D cartesian mesh is made of $50 \times 250$ cells. At time $t=0 \mathrm{~s}$, a stationary vortex similar to the one already introduced in subsection (5.3) is present at $\left(x_{0}, y_{0}\right)=(7,1)$. Simultaneously, a smooth but steep pressure pulse is injected along all the pipe section at $x_{1}=5$ m. Such a pulse reads:

$$
\begin{aligned}
p & =p_{0}\left(1+9 \times e^{-\left(x-x_{1}\right)^{2} / 2 \delta^{2}}\right), \delta=1.7 \times 10^{-2} \mathrm{~m}, \\
p_{0} & =1 \text { bar. }
\end{aligned}
$$

The value of the parameter $\delta$ is chosen such that, initially, the width of the acoustic pressure pulse is smaller than the length of one cell of the considered mesh. Outside the vortex, the other initial conditions are $\left(u_{0}, v_{0}\right)=(0,0)$ and $\rho_{0}=1 \mathrm{~kg} \cdot \mathrm{m}^{-3}$. Transmissive boundary conditions hold on the left and right borders of the computational domain. The top and bottom borders are considered to be walls.

At $t=0 \mathrm{~s}$, the initial pressure pulse generates symmetric acoustic waves associated with a rise of the velocity behind their front line. In these regions the Mach number is about 0.4 by contrast with the maximal Mach number inside the vortex which is approximately $10^{-2}$ showing the wide range of Mach number values involved simultaneously in the present case. Within an acoustic time-scale, the acoustic wave has interacted and then crossed the vortex.

As previously mentioned, the adaptivity of the splitting is assessed in this test-case. Indeed, one would like to check that the proposed FVS approach is able to locally decouple the convective and pressure parts in low Mach number regions and retrieve the overall Euler system when the Mach number rises. In the low Mach number regions, the corrections holding on the pressure part of the flux should allow to be accurate with respect to the incompressible solutions. In regions where the Mach number scales as $O(1)$, the classical upwinding performed on the convective flux should allow to capture the fast acoustic wave dynamics.

Figure 10 displays the propagation of the acoustic pressure wave at the beginning, half and final time of the simulation. The present approach is compared to the HLLC scheme. It turns out that the proposed scheme manages to capture and follow the compressible wave. Because the maximal flow Mach number is about 0.4 , the pressure part of the splitting does not completely cancel and the anti-diffusion brought by low-Mach corrections contribute to preserve the acoustic wave profile.

Finally, in Figure 11, the flow Mach number is plotted using the scaling of the Gresho vortex. One can observe that after having interacted with the acoustic wave, the structure of the stationary vortex is better preserved in the case of the present FVS approach than with the HLLC scheme. 
(a) HLLC scheme

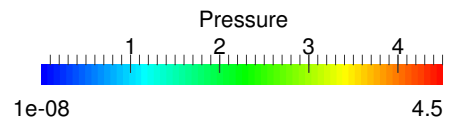

(1)
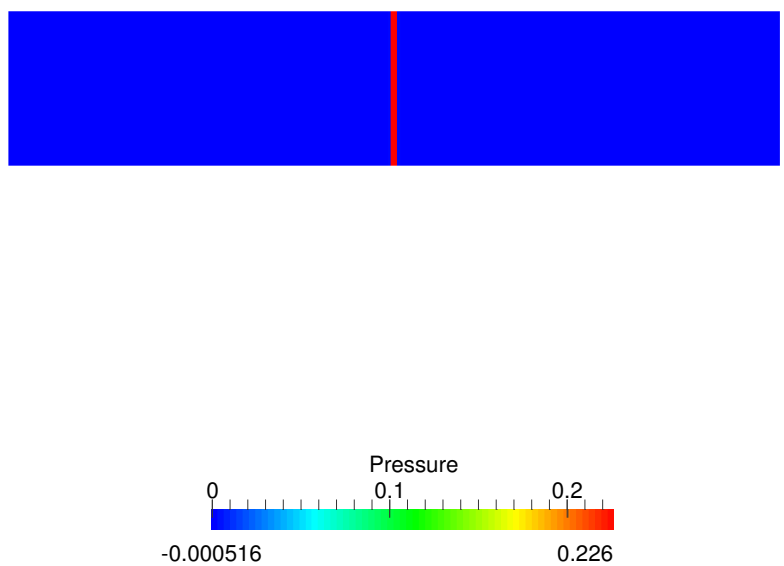

(2)
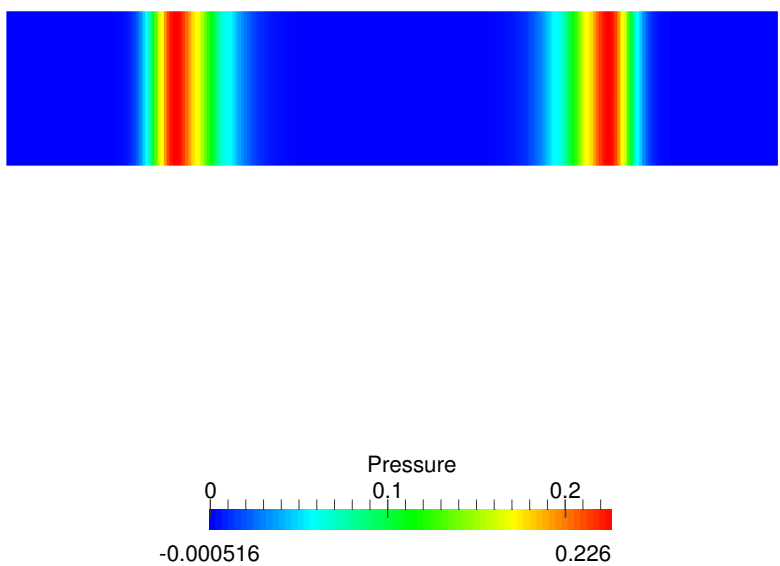

(3)

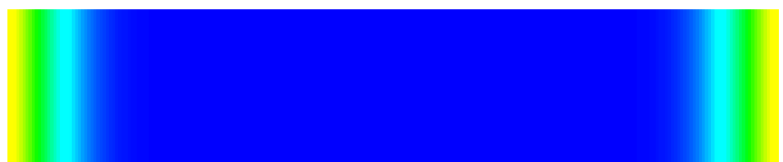

(b) present FVS scheme
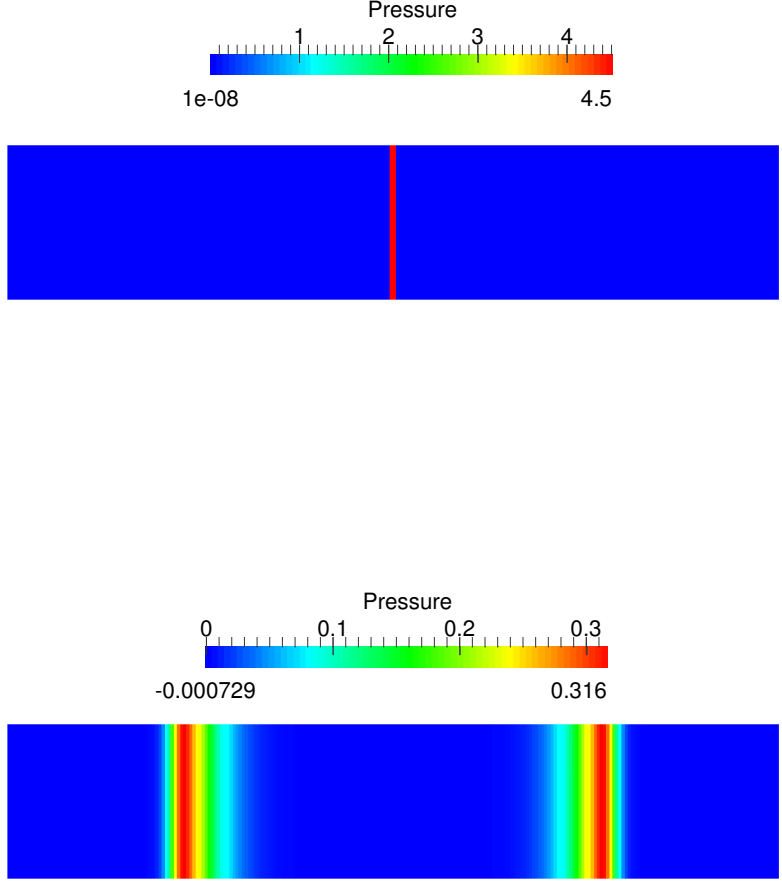

Figure 10: Acoustic pressure waves at time (1) $t=0 \mathrm{~s}$, (2) $t=6 \times 10^{-3} \mathrm{~s}$ and (3) $t=1.2 \times 10^{-2} \mathrm{~s}$, mesh made of $50 \times 250$ cells, Courant number $C=0.45$; with (a) HLLC scheme, (b) the present approach 
(a) HLLC scheme

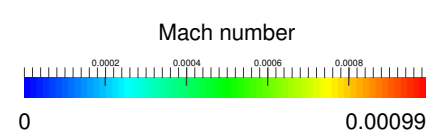

(1)

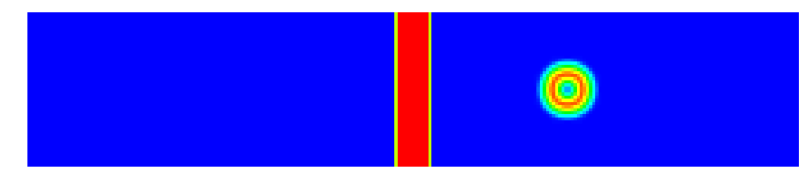

Mach number

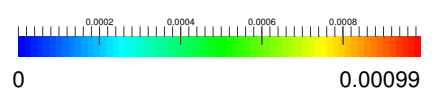

(2)
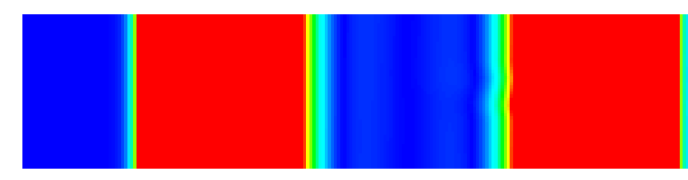

Mach number

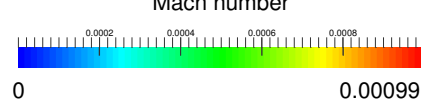

(3)
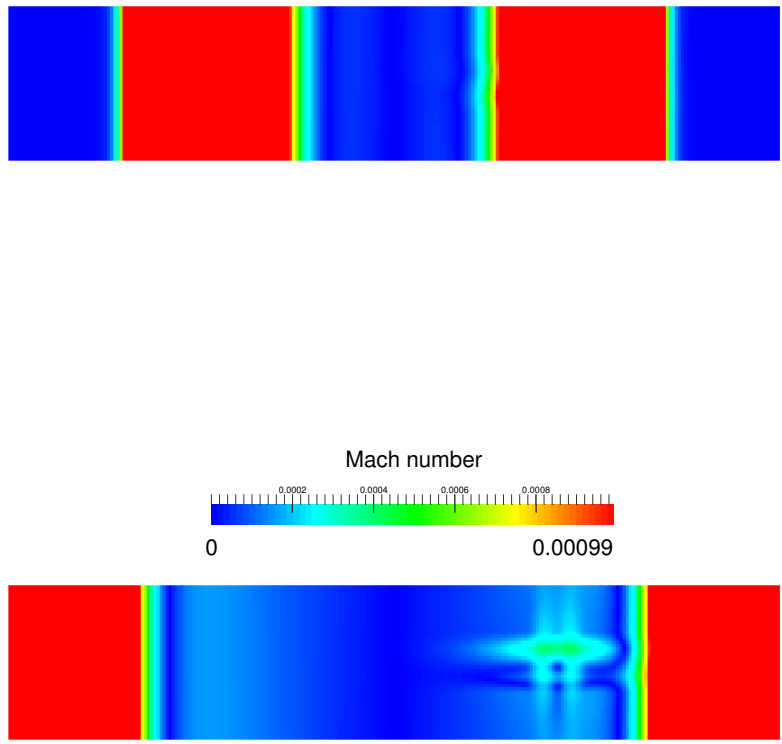

(b) present FVS scheme
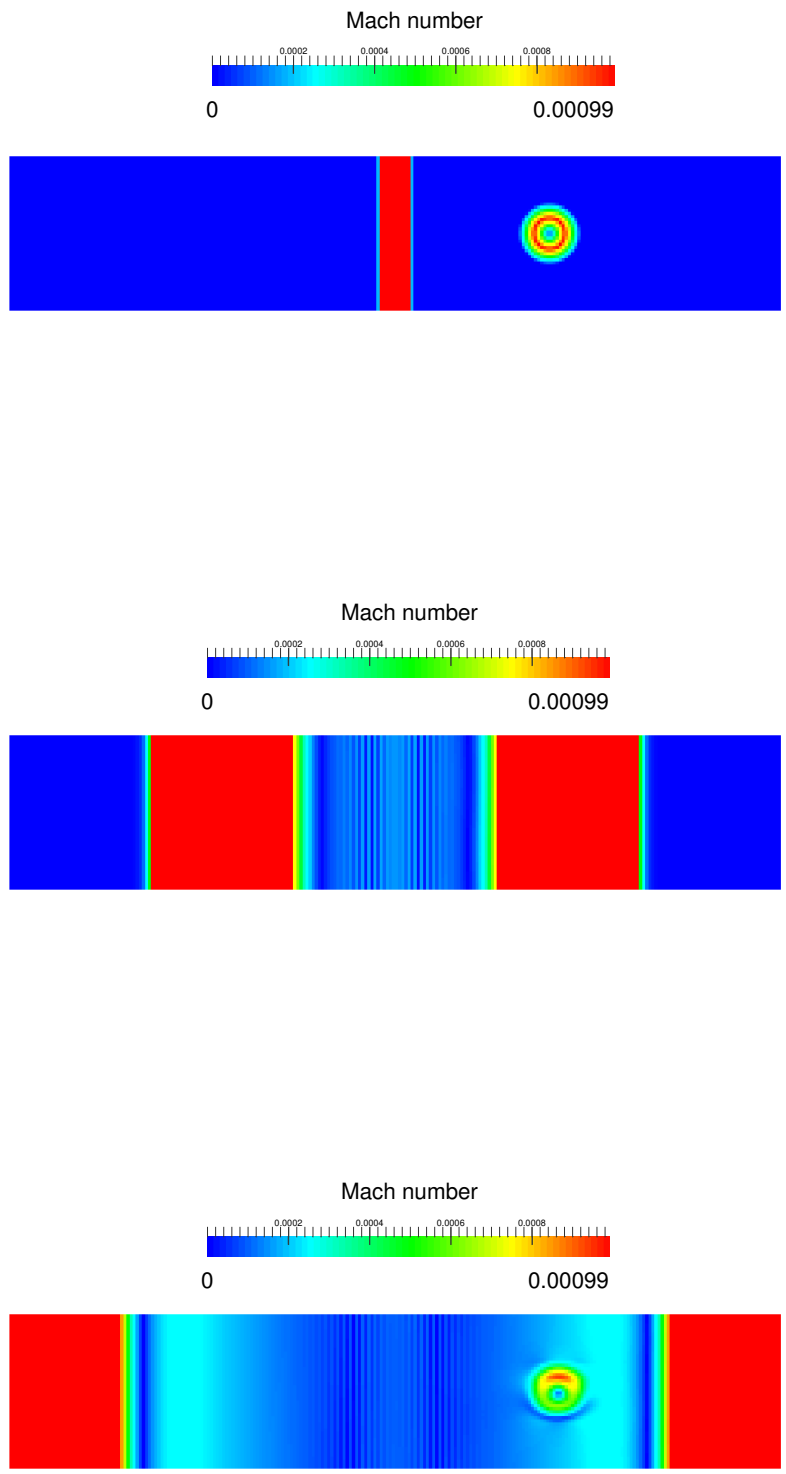

Figure 11: Mach number at time $(1) t \approx 10^{-6} \mathrm{~s}$, mesh made of $50 \times 250$ cells, Courant number $C=0.45$; with (a) HLLC scheme, (b) the present approach 


\section{Conclusions and perspectives}

In the present work, a time-explicit Flux-Vector Splitting solver has been proposed. Its construction relies on a dynamic splitting of the Euler system flux highlighting a convective and a pressure part. The dynamic aspect of the splitting stems from a unique parameter $\mathscr{E}_{0}$ which follows the space-and-time variations of the Mach number across the computational domain. In low-Mach number regions, $\mathscr{E}_{0}$ tends towards zero, and the whole acoustic wave production is transfered to the pressure part of the splitting. A HLLC-like method applied to the underlying acoustic subsystem is then used to discretize the pressure flux. Besides, in order to handle the low-Mach accuracy issue inherent to any Godunov-like scheme, low-Mach corrections holding on the momentum and the energy components of the pressure flux have been added. In sonic or highly subsonic regions, $\mathscr{E}_{0}$ is equal to one and the entire compressible Euler flux is retrieved in the convective part of the splitting. In that case a simple upwind scheme, based on the intermediate velocity and pressure produced by the pressure part, is used to discretize the convective flux.

A Sod's shock tube has allowed to verify that the proposed approach is able to capture fast compressible shock and rarefaction waves. Besides, it can also preserve exactly stationary contact waves. When tested against stationary incompressible test cases, the low-Mach corrections injected in the pressure flux of the present method contribute to capture the appropriate incompressible solutions. Besides, contrary to other time-explicit solvers, the proposed scheme seems to be stable under a classical CFL condition based on the Euler eigenvalues. However, let us recall that, the formal analysis holding on the invariance principle of the discrete incompressible phase space suggests that a lowMach correction should also be applied to the non-centered part of the intermediate velocity in the energy component of the pressure flux. Such a correction has been voluntarily avoided as it disturbed the stability of the present method. Hence, a first perspective to the present work could be to carry out a stability study based on a linearized version of the splitting in order to determine the optimal scaling of $\mathscr{E}_{0}$ with respect to the different low-Mach corrections. An other perspective could be to derive an implicit-explicit version of the present FVS scheme. Indeed the time-implicit integration of the pressure part of the flux might contribute to increase the range of values of the Courant number for which the method is stable. Eventually, one could also check that such an implicit-explicit version of the scheme is asymptotically preserving towards the Euler incompressible system.

\section{Acknowledgement}

The first author received a financial support through the EDF-CIFRE contract 0561-2015. Computational facilities were provided by EDF. The code Europlexus, used to perform the simulations, is co-owned by the French Commissariat à l'énergie atomique et aux énergies alternatives (CEA) and by the European Commission. Électricité de France (EDF) is involved as a major partner of the consortium established for Europlexus software development (http://www-epx.cea.fr/). 


\section{References}

[1] C. Hirsch, Numerical Computation of Internal and External Flows, J. Wiley and Sons, New-York, 1988.

[2] R. J. LeVeque, Finite Volume Methods for Hyperbolic Problems, Cambridge University Press, 2002.

[3] E. F. Toro, Riemann Solvers and Numerical Methods for Fluid Dynamics. A Practical Introduction, 3d edition, Springer, 2009.

[4] P. L. Roe, Approximate Riemann solvers, parameter vectors, and difference schemes, J. Comput. Phys. 43 (1981) $357-372$.

[5] E. F. Toro, M. Spruce, W. Speares, Restoration of the contact surface in the HLL-Riemann solver, Shock Waves 4 (1) (1994) $25-34$.

[6] J. L. Steger, R. F. Warming, Flux Vector Splitting of the Inviscid Gasdynamic Equations with Application to Finite Difference Methods, J. Comput. Phys. 40 (2) (1981) 263-293.

[7] M.-S. Liou, C. J. Steffen, A New Flux Splitting Scheme, J. Comput. Phys. 107 (1) (1993) 23-39.

[8] G.-C. Zha, E. Bilgen, Numerical solutions of Euler equations by using a new flux vector splitting scheme, Int. J. Numer. Methods Fluids 17 (1993) 115-144.

[9] E. F. Toro, M. E. Vázquez-Cendón, Flux splitting schemes for the Euler equations, Comput. Fluids 70 (2012) 1-12.

[10] J. C. Mandal, V. Panwar, Robust HLL-type Riemann solver capable of resolving contact discontinuity, Comput. Fluids 63 (2012) $148-164$.

[11] H. Guillard, C. Viozat, On the behavior of upwind schemes in the low Mach number limit, Comput. Fluids 28 (1999) 63-86.

[12] H. Guillard, A. Murrone, On the behavior of upwind schemes in the low Mach number limit: II Godunov type schemes, Comput. Fluids 33 (2004) 655-675.

[13] F. Rieper, On the behaviour of numerical schemes in the low Mach number regime, Ph.D. thesis, Brandenburgischen Technischen Universitt Cottbus (2008).

[14] S. Dellacherie, Analysis of Godunov type schemes applied to the compressible Euler system at low Mach number, J. Comput. Phys. 229 (4) (2010) 978-1016.

[15] S. Dellacherie, J. Jung, P. Omnes, P.-A. Raviart, Construction of modified Godunov-type schemes accurate at any Mach number for the compressible Euler system, Math. Models Methods Appl. Sci. 26 (13) (2016) 2525-2615.

[16] S. Dellacherie, P. Omnes, F. Rieper, The influence of cell geometry on the Godunov scheme applied to the linear wave equation, J. Comput. Phys. 229 (2010) 5315-5338.

[17] F. Rieper, A low-Mach number fix for Roe's approximate Riemann solver, J. Comput. Phys. 230 (2011) $5263-5287$.

[18] P. Fillion, A. Chanoine, S. Dellacherie, A. Kumbaro, FLICA-OVAP: A new platform for core thermalhydraulic studies, Nuclear Eng. Design 241 (2011) 4348-4358.

[19] K. Oßwald, A. Siegmund, P. Birken, V. Hannemann, A. Meister, L2Roe: a low dissipation version of Roe's approximate Riemann solver for low Mach numbers, Int. J. Numer. Methods Fluids 81 (2016) 71-86.

[20] P. Bruel, S. Delmas, J. Jung, V. Perrier, A low Mach correction able to deal with low Mach acoustics, J. Comput. Phys. (2018) Accepted for publication.

[21] M.-S. Liou, A sequel to AUSM: AUSM+, J. Comput. Phys. 129 (2) (1996) 364-382.

[22] M.-S. Liou, A sequel to AUSM, Part II: AUSM+'-up for all speeds, J. Comput. Phys. 214 (1) (2006) 137-170.

[23] R. Baraille, Développement de schémas numériques adaptés à l'hydrodynamique ; méthode numérique à pas fractionnaires, application aux calculs d'écoulements hypersoniques réactifs non visqueux ; formulation du schéma MUSCL de Van Leer pour une configuration axisymétrique., Ph.D. thesis, Université Bordeaux I (1991).

[24] R. Baraille, G. Bourdin, F. Dubois, A. Y. Le Roux, Une version à pas fractionnaires du schéma de Godunov pour l'hydrodynamique, C.R. Acad. Sci. Paris 314 (1992) 147-152.

[25] D. Iampietro, F. Daude, P. Galon, J.-M. Hérard, A Mach-Sensitive Splitting Approach for Euler-like Systems, ESAIM Math. Model. Numer. Anal 52 (1) (2018) 207-253.

[26] P. Batten, N. Clarke, C. Lambert, D. M. Causon, On the choice of wavespeeds for the HLLC Riemann solver, SIAM J. Sci. Comput. 18 (6) (1997) 1553-1570.

[27] E. F. Toro, A. Chakraborty, The development of a Riemann solver for the steady supersonic Euler equations, The Aeronautical Journal 98 (979) (1994) 325-339.

[28] F. Daude, P. Galon, A Finite-Volume approach for compressible single- and two-phase flows in flexible pipelines with fluid-structure interaction, J. Comput. Phys. 362 (C) (2018) 375-408.

[29] F. Daude, A. S. Tijsseling, P. Galon, Numerical investigations of water-hammer with column-separation induced by vaporous cavitation using a one-dimensional Finite-Volume approach, J. Fluids Struct. 83 (2018) 91-118.

[30] D. Iampietro, F. Daude, P. Galon, J.-M. Hérard, A Mach-sensitive implicit-explicit scheme adapted to compressible multi-scale flows, J. Comput. Appl. Math. 340 (2018) 122-150.

[31] C. Chalons, M. Girardin, S. Kokh, An all-regime Lagrange-Projection like scheme for the gas dynamics equations on unstructured meshes, Commun. Comput. Phys. 20 (1) (2016) 188-233.

[32] S. Dellacherie, Checkerboard modes and wave equation, proceedings of ALGORITMY (2009) 71-80.

[33] C. Chalons, M. Girardin, S. Kokh, An all-regime Lagrange-Projection like scheme for 2D homogeneous models for two-phase flows on unstructured meshes, J. Comput. Phys. 335 (2017) 885-904.

[34] Joint Research Centre (JRC), Commissariat à l'énergie atomique et aux énergies alternatives (CEA), Europlexus user's manual, http://europlexus.jrc.ec.europa.eu/ (2018).

[35] G. A. Sod, A survey of several finite difference methods for systems of nonlinear hyperbolic conservation law, J. Comput. Phys. 27 (1) (1978) $1-31$.

[36] P. M. Gresho, On the theory of semi-implicit projection methods for viscous incompressible flow and its implementation via a finite element method that also introduces a nearly consistent mass matrix. I: Theory, Int. J. Numer. Methods Fluids 11 (5) (1990) 587-620.

[37] P. M. Gresho, S. T. Chan, On the theory of semi-implicit projection methods for viscous incompressible flow and its implementation via a finite element method that also introduces a nearly consistent mass matrix. II: Implementation, Int. J. Numer. Methods Fluids 11 (5) (1990) 621-659. 
[38] F. Miczek, F. K. Röpke, P. V. Edelmann, New numerical solver for flows at various Mach numbers, Astronomy and Astrophysics 576 (2015) A50. 